OPEN ACCESS

Edited by:

Marijn Lijffijt,

Baylor College of Medicine,

United States

Reviewed by:

Jay McLaughlin,

University of Florida, United States

Ryan K. Bachtell,

University of Colorado Boulder,

United States

*Correspondence:

Claire Gaveriaux-Ruff

gaveriau@igbmc.fr

†These authors have contributed equally to this work

Specialty section:

This article was submitted to

Psychopharmacology,

a section of the journal

Frontiers in Psychiatry

Received: 17 July 2018 Accepted: 10 December 2018

Published: 04 January 2019

Citation:

Maduna T, Audouard E, Dembélé D, Mouzaoui N, Reiss D, Massotte D and

Gaveriaux-Ruff C (2019) Microglia Express Mu Opioid Receptor: Insights From Transcriptomics and Fluorescent

Reporter Mice.

Front. Psychiatry 9:726

doi: 10.3389/fpsyt.2018.00726

\title{
Microglia Express Mu Opioid Receptor: Insights From Transcriptomics and Fluorescent Reporter Mice
}

\author{
Tando Maduna ${ }^{1,2,3,4 \dagger}$, Emilie Audouard ${ }^{1,2,3,4 \dagger}$, Doulaye Dembélé ${ }^{1,2,3,4}$, \\ Nejma Mouzaoui 1,2,3,4,5, David Reiss ${ }^{1,2,3,4}$, Dominique Massotte ${ }^{6}$ and \\ Claire Gaveriaux-Ruff ${ }^{1,2,3,4,5 *}$
}

${ }^{1}$ Institut de Génétique et de Biologie Moléculaire et Cellulaire, Illkirch, France, ${ }^{2}$ Université de Strasbourg, Illkirch, France, ${ }^{3}$ Centre National de la Recherche Scientifique, Illkirch, France, ${ }^{4}$ Institut National de la Santé et de la Recherche Médicale, IIIkirch, France, ${ }^{5}$ Ecole Supérieure de Biotechnologie de Strasbourg, Illkirch, France, ${ }^{6}$ CNRS UPR3212, Institut des Neurosciences Cellulaires et Intégratives, Centre National de la Recherche Scientifique, Université de Strasbourg, Strasbourg, France

Background: Microglia activation contributes to chronic pain and to the adverse effects of opiate use such as analgesic tolerance and opioid-induced hyperalgesia. Both mu opioid receptor (MOR) encoded by Oprm1/OPRM1 gene and toll like receptor 4 (TLR4) have been reported to mediate these morphine effects and a current question is whether microglia express the Oprm1 transcript and MOR protein. The aim of this study was to characterize Oprm1-MOR expression in naive murine and human microglia, combining transcriptomics datasets previously published by other groups with our own imaging study using the Cx3cr1-eGFP-MOR-mCherry reporter mouse line.

Methods: We analyzed microglial Oprm1/OPRM1 expression obtained from transcriptomics datasets, focusing on ex vivo studies from adult wild-type animals and adult post-mortem human cerebral cortex. Oprm1, as well as co-regulated gene sets were examined. The expression of MOR in microglia was also investigated using our novel fluorescent Cx3cr1-eGFP-MOR-mcherry reporter mouse line. We determined whether CX3cR1-eGFP positive microglial cells expressed MOR-mCherry protein by imaging various brain areas including the Frontal Cortex, Nucleus Accumbens, Ventral Tegmental Area, Central Amygdala, and Periaqueductal Gray matter, as well as spinal cord.

Results: Oprm1 expression was found in all 12 microglia datasets from mouse whole brain, in 7 out of 8 from cerebral cortex, 3 out of 4 from hippocampus, 1 out of 1 from striatum, and 4 out of 5 from mouse or rat spinal cord. OPRM1 was expressed in 16 out of 17 microglia transcriptomes from human cerebral cortex. In Cx3cr1-eGFP-MOR-mCherry mice, the percentage of MOR-positive microglial cells ranged between 35.4 and 51.6\% in the different brain areas, and between 36.8 and $42.4 \%$ in the spinal cord. 
Conclusion: The comparative analysis of the microglia transcriptomes indicates that Oprm1/OPRM1 transcripts are expressed in microglia. The investigation of Cx3cr1eGFP-MOR-mCherry mice also shows microglial expression of MOR proteinin the brain and spine. These results corroborate functional studies showing the actions of MOR agonists on microglia and suppression of these effects by MOR-selective antagonists or MOR knockdown.

Keywords: microglia, opioid receptor, mu, transcriptomics, gene clusters, fluorescent reporter mice, analgesic tolerance, opioid-induced hyperalgesia

\section{INTRODUCTION}

Activation of the mu opioid receptor (MOR), encoded by the Oprm1/OPRM1 gene in rodents and humans, respectively $(1,2)$, mediates opioid analgesia and the adverse consequences of opioid use $(3,4)$. Glial cells and in particular microglia are known to contribute to chronic pain (5) as well as to opioid tolerance and opioid-induced hyperalgesia $(\mathrm{OIH})(6-8)$. However, whether microglia express Oprm1 and whether microglial Oprm1 would have a role in chronic pain and other opioid effects remains to be demonstrated. Most studies reporting MOR expression or function in microglia have been performed on cultured microglia (9-18). It has been shown however that gene expression profiles differ between microglia in culture and adult mouse microglia in vivo (19) and therefore the demonstration of MOR expression in cultured microglia does not allow to conclude for MOR expression in adult microglia in vivo. Horvath et al. (20) have shown MOR expression in rat spinal microglia in vivo by immunohistochemistry. However, two other studies have contradicted these findings by showing a lack of MOR expression in spinal cord microglia $(21,22)$. Thus, whether microglia in adults express MOR still remains an unsolved question. Specifically, Corder et al. present considerable evidence that argues against the expression of MOR messenger and protein in mouse spinal cord microglia. Their findings are further strengthened by transcriptomic analyses which show a lack in co-expression of Oprm1 mRNA with microglial markers (21). However, critical analysis and commentary on these interesting results is not possible yet due to the limited access of the datasets used for transcriptomic analysis. Therefore, whether Oprm1/OPRM1 are expressed by microglia remains a matter of debate that should be further investigated. To date, there are no published studies focusing on the analysis of a large series of transcriptomic datasets for Oprm1 expression in microglia that would allow assessing, unambiguously, Oprm1 expression in microglia in vivo. In addition, OPRM1/MOR expression in human microglia is yet to be fully characterized. For this purpose, we have used novel approaches to characterize Oprm1/OPRM1

Abbreviations: BDNF, brain-derived neurotrophic factor; CeA, central amygdala; CTAP, D-Phe-Cys-Tyr-D-Trp-Arg-Thr-Pen-Thr-NH2; eGFP, enhanced green fluorescent protein; FCx, frontal cortex; KO, knockout; MA, microarray; Marsseq, massively parallel Single-cell RNA-seq; MOR, mu opioid receptor; NAcc, nucleus accumbens; $\mathrm{OIH}$, opioid-induced hyperalgesia; PAG, periaqueductal gray; PBS, phosphate buffer baline; PFA, paraformaldehyde; RNA-seq, RNA-sequencing; TLR, toll like receptor; VTA, ventral tegmental area. expression in microglia based on transcriptomics and have used fluorescent reporter mice to characterize MOR expression in vivo.

A number of laboratories have generated transcriptomics datasets for microglia that can be used for analyzing gene expression profiles $(23,24)$ as well as for investigating microglia physiology and their responses in disease $(25,26)$. We have analyzed published datasets from microarray (MA) and RNAsequencing (RNA-seq) studies performed on rodent and human microglia for Oprm1 and OPRM1 gene expression. We have completed this analysis by imaging microglial MOR using a novel double fluorescent Cx3cr1-eGFP-MOR-mCherry mouse line. The chemokine receptor CX3CR1 is a specific marker for phagocytic cells and labels specifically microglia and macrophages in the nervous system of naive animals (27-29). The Cx3cr1-eGFP mouse line was originally used to explore CX3CR1 function (30) then later to map the fate of tissue macrophages including microglia $(28,29)$. A reporter knock-in mouse line for MOR, the MOR-mCherry line, allows to map the distribution of MOR-expressing cells in mice using fluorescence imaging (31). In order to localize MOR protein in microglia, we have bred these two lines together to generate the $\mathrm{Cx} 3 \mathrm{cr} 1$ eGFP-MOR-mCherry mouse line. Thus, we have analyzed MOR expression in various brain regions implicated in chronic pain or chronic opiate effects as well as the spinal cord in control non-pathological conditions. In addition, as sex differences are an important factor for chronic pain (32-34) and greatly impact the microglial contribution to pain $(35,36)$, we investigated Oprm1/OPRM1-MOR presence in microglia from both females and males.

\section{MATERIALS AND METHODS}

\section{Transcriptomics Analyses}

As microglia develop after birth until post-natal day 15, followed by stabilization of microglial numbers (37-39), we focused the Oprm1 expression analysis on microglia from juvenile-adult wild-type naïve rodent and juvenile-adult humans with no reported pain phenotype. The datasets used and related information including the pathology of patients from whom microglia were collected, are indicated in Supplementary Tables 1-4. The selection criteria for which datasets to include in the present study were as follows: normalized mouse datasets included in the database recently published by Friedman et al. (40) (see Data S2 in Friedman et al.) and additional normalized datasets containing Oprm1 
in their gene list; from wild-type mice aged 0.5 months and older. Microglia datasets from mouse whole brain were from the studies by Wang et al. (41), Verheijden et al. (42), Poliani et al. (43), Erny et al. (44), Szulzewsky et al. (45), Pyonteck et al. (46), Bruttger et al. (47), Lavin et al. (48), Bennett et al. (49), Gosselin et al. (50), Krasemann et al. (51), and Zhao et al. (52). Microglia datasets from mouse brain areas were from Orre et al. (53), Arumugan et al. (54), Grabert et al. (55), Friedman et al. (40), Srinivasan et al. (56), Zhang et al. 2014 (57), and Matcovitch-Natan et al. (58). Microglia datasets from rodent spinal cord were from Chiu et al. (59), Denk et al. (60), Noristani et al. (61), Matcovitch-Natan et al. (58), and Jokinen et al. (62). Information on these datasets including publication authors and year, accession number, mouse or rat strain, sex, age, dissociation, and microglia isolation methods, and genomics assay are given in Supplementary Tables 1-4.

Human datasets comprised those published by Zhang et al. (63) and contained in Friedman's Supplementary Table 2 as well as normalized datasets recently reported by Gosselin et al. (64) and Galatro et al. (65) as described in Supplementary Table 4. Normal cortical regions were resected from patients with diseases described in column F. Among the 19 datasets by Gosselin et al. we selected 10 datasets derived from individuals aged 13 and older. Among the 39 datasets from adult individuals by Galatro et al. we selected 8 datasets derived from samples collected at a maximum delay of $10 \mathrm{~h}$ post-mortem. Information on these datasets including publication authors and year, accession number, sex, age, cortex area, dissociation, and microglia isolation methods, and genomics assay are given in Supplementary Table 4.

In addition to our main focus set, (A) showing only the expression levels of Oprm1 gene transcript from purified microglia, three other groups of gene clusters were formed: (B) Myeloid cells, (C) Activation patterns and (D) Neurons and Astrocytes. The list of genes contained in each of the gene clusters of $\mathrm{B}, \mathrm{C}$, and $\mathrm{D}$ was formed using the gene lists defined in Friedman et al. (40) and filtered to exclude the few genes expressed by any other cell type. The Myeloid gene clusters (B) include the Microglia, the Macrophage, and the Neutrophil-Monocytes gene clusters, respectively. The activation pattern clusters (C) comprise the Interferon-related, Proliferation-related, LPS-related and Neurodegeneration-related gene clusters. The Neurons and Astrocyte gene clusters (D) are composed of the Neuronassociated, Excitatory neuron-associated, GABAergic neuronassociated, and Astrocyte-associated genes clusters.

The logarithm scale two $(\log 2)$ transformation was applied to each data sample values and 1 was added to expression values to avoid indetermination for zero count reads. For each data sample, the first (25th percentile), the second (median), and the third quantile (75th percentile) values were computed. The $\log 2$ values for the Oprm1 transcript were also obtained for each dataset. The $\log 2$ values associated with each gene subset of B, C, and $\mathrm{D}$ categories were isolated for each sample and an average value was calculated for each subset. The R environment (version 3.5.0) was used to create the basic figures. The vioplot $\mathrm{R}$ package was used to obtain a violin plot like contour for the $\log 2$ values of each data sample. The $\log 2$ values and the 25 th, 75 th percentile and median values were superimposed on the violin plots using different colors as shown in Figures 1-4.

For determining correlations between Oprm1/OPRM1 expression and the expression of the gene clusters described above, $\mathrm{z}$-scores transformations $(1+\log 2$ values) were calculated from the mean expression level of each cluster for each dataset included in the study. The Orre et al. (53) mouse cortex dataset and the S037 dataset of the Gosselin et al. study (64) were removed from the analysis as they varied substantially from all other datasets within their group. The correlation analysis for human datasets included all dataset except for S037 set.

\section{Cx3cr1-eGFP-MOR-mCherry Mouse Line Animals and Ethics Statement}

The animals were housed under standard light, temperature, and humidity conditions ( $12 \mathrm{~h}$ light-dark cycle, $21 \pm 1{ }^{\circ} \mathrm{C}, 55 \pm 10 \%$ humidity) with food and water ad libitum. Brains and spinal cords were collected from male and female mice aged between 5 and 15 weeks. All experiments were conducted respecting the European Communities Council Directives of 22 September 2010 (directive 2010/63/UE) under the guidelines of the Committee for Research and Ethical issues of IASP published in PAIN, 1983; 16:109-110, and were approved by the local ethical committee (Com'Eth d'Ethique pour l'Expérimentation Animale IGBMCICS, license $\mathrm{N}^{\circ} 17$ ) with the agreement number 00876-02.

\section{Cx3cr1-eGFP-MOR-mCherry Mice}

The Cx3cr1-eGFP-MOR-mCherry mouse line was generated by crossing Cx3Cr1-eGFP mice (29) and MOR-mCherry mice (31) to obtain viable heterozygous animals. These were intercrossed to generate homozygous $\mathrm{Cx} 3 \mathrm{Cr} 1-\mathrm{eGFP}-\mathrm{MOR}$ mCherry mice that are fertile and develop normally. Genotyping was performed by PCR to detect both Cx3Cr1-GFP sequence (35 cycles at $94^{\circ} \mathrm{C}$ for $30 \mathrm{~s}, 65.5^{\circ} \mathrm{C}$ for $30 \mathrm{~s}$, and $72^{\circ} \mathrm{C}$ for $2 \mathrm{~min}$ ) using the following primer sequences: $\mathrm{Cx} 3 \mathrm{Cr} 1-\mathrm{Fwd}: 5^{\prime}$ TTCACGTTCGGTCTGGTGGGAAATC-3', Cx3Cr1- Rev:

5'-TTCCTAGTGGAGCTAGGGTCGGGG-3', eGFP-Fwd:

5'-GATCACTCTCGGCATGGACG-3', and MOR-mCherry sequence $\left(35\right.$ cycles at $94^{\circ} \mathrm{C}$ for $1 \mathrm{~min}, 63^{\circ} \mathrm{C}$ for $1 \mathrm{~min}$, and $72^{\circ} \mathrm{C}$ for $1 \mathrm{~min}$ ) using a forward primer located on exon four of Oprm1 gene, -Fwd: 5'-TGACGTGACATGCAGTTGAGATTT$3^{\prime}$ and a reverse primer located in the $3^{\prime}$ UTR region, Rev: $5^{\prime}$ TCCCACAAACCCTGACAGCAAC- $3^{\prime}$. Both female and male Cx3cr1-eGFP-MOR-mCherry mice were analyzed for MOR expression in microglia ( 3 animals per sex).

\section{Tissue Preparation}

Mice were deeply anesthetized with intraperitoneal administration of $100 / 5 \mathrm{mg} / \mathrm{kg}$ ketamine/xylazine (Virbac, Carros, France; Rompun, Bayer, La Garenne Colombes, France) and were intracardially perfused with $4 \%$ Paraformaldehyde (PFA) in Phosphate Buffer Saline (PBS) solution (4\%PFA/PBS). The whole brain and spinal cord were isolated and post-fixed overnight at $4^{\circ} \mathrm{C}$ in $4 \% \mathrm{PFA} / \mathrm{PBS}$. Samples were rinsed three times in PBS and cryoprotected in a sucrose/PBS gradient (10, 20 , and $30 \%$ ) for $24 \mathrm{~h}$ each. Tissues were embedded in OCT 
A

.

Myeloid cells

Microglia

Macrophages

Neutrophil-monocyte

c

Activation patterns

Interferon

Proliferation

LPS

Neurodegeneration

D Neurons and Astrocytes

Neurons

Excitatory

GABAergic

Astrocytes
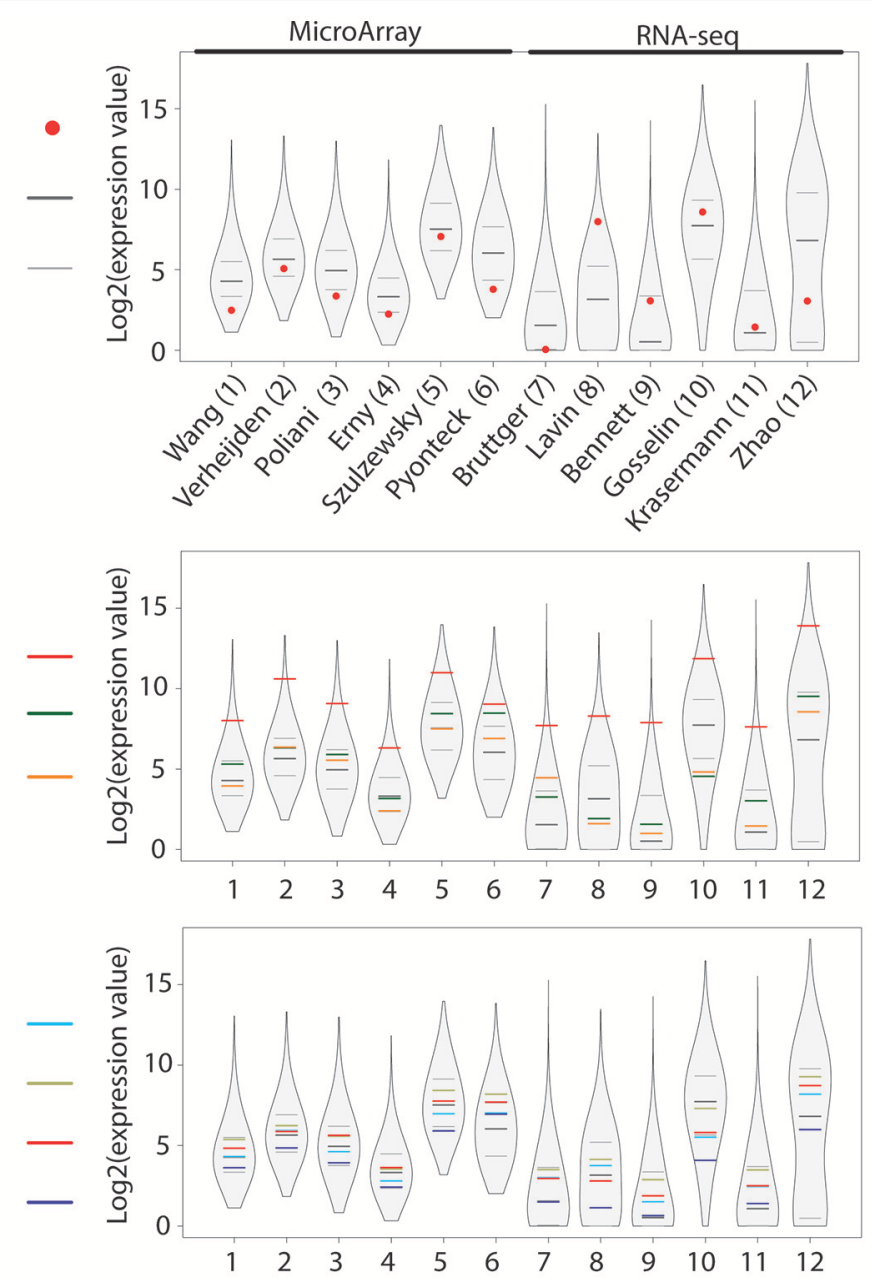

$-$

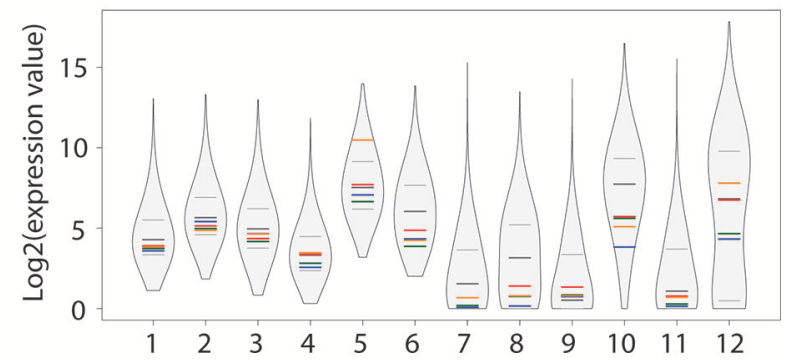

FIGURE 1 | Oprm1 and gene clusters expression in mouse whole brain microglia datasets. Expression levels of the genes identified in each dataset are represented in violin plots demonstrating the median, as well as the 25th and 75th quartiles. Datasets are represented by name of the first author in (A), which are denoted numerically in (B-D). (A) Expression of Oprm1 is below the median in purified microglia assayed with MA, and fluctuates above and below the median in purified microglia assayed with RNA-seq. (B) Expression values of the Myeloid cell clusters demonstrate a high expression of Microglia-related genes which are in the 75th quartile in all the datasets analyzed. (C) Expression values of the Activation pattern clusters are below the 75th quartile in all but one dataset (dataset \#6). (D) Expression values of the Neurons and Astrocytes clusters are below the median in 5 out 6 of the datasets derived from MA assays, whereby the Astrocyte-related genes have expression values above the 75th quartile in dataset \#5. When assayed with RNA-seq, the Astrocyte-related genes are below the median, except for dataset \#12, where the values are above the median.

(Tissue Tek, Sakura Fine Technical, Torrance) and coronally (brain) or transversally (spinal cord) sectioned at $30 \mu \mathrm{m}$ on Superfrost microscope slides using a cryostat (Leica CM3050S). Cryosections were dried at room temperature and then stored at $-20^{\circ} \mathrm{C}$ before imaging.

\section{Immunohistochemistry and Quantifications}

For immunohistochemistry, cryosections were rehydrated with PBS and were then incubated in a blocking solution (10\% horse serum/0.1\% Triton X-100/PBS) for $30 \mathrm{~min}$ at room temperature. The sections were incubated with the primary antibodies diluted 
Oprm1

Oprm1

Median

Quartiles

B

Myeloid cells

Microglia

Macrophages

Neutrophil-monocyte

C

Activation patterns

Interferon

Proliferation

LPS

Neurodegeneration

D Neurons and Astrocytes

Neurons

Excitatory

GABAergic

Astrocytes
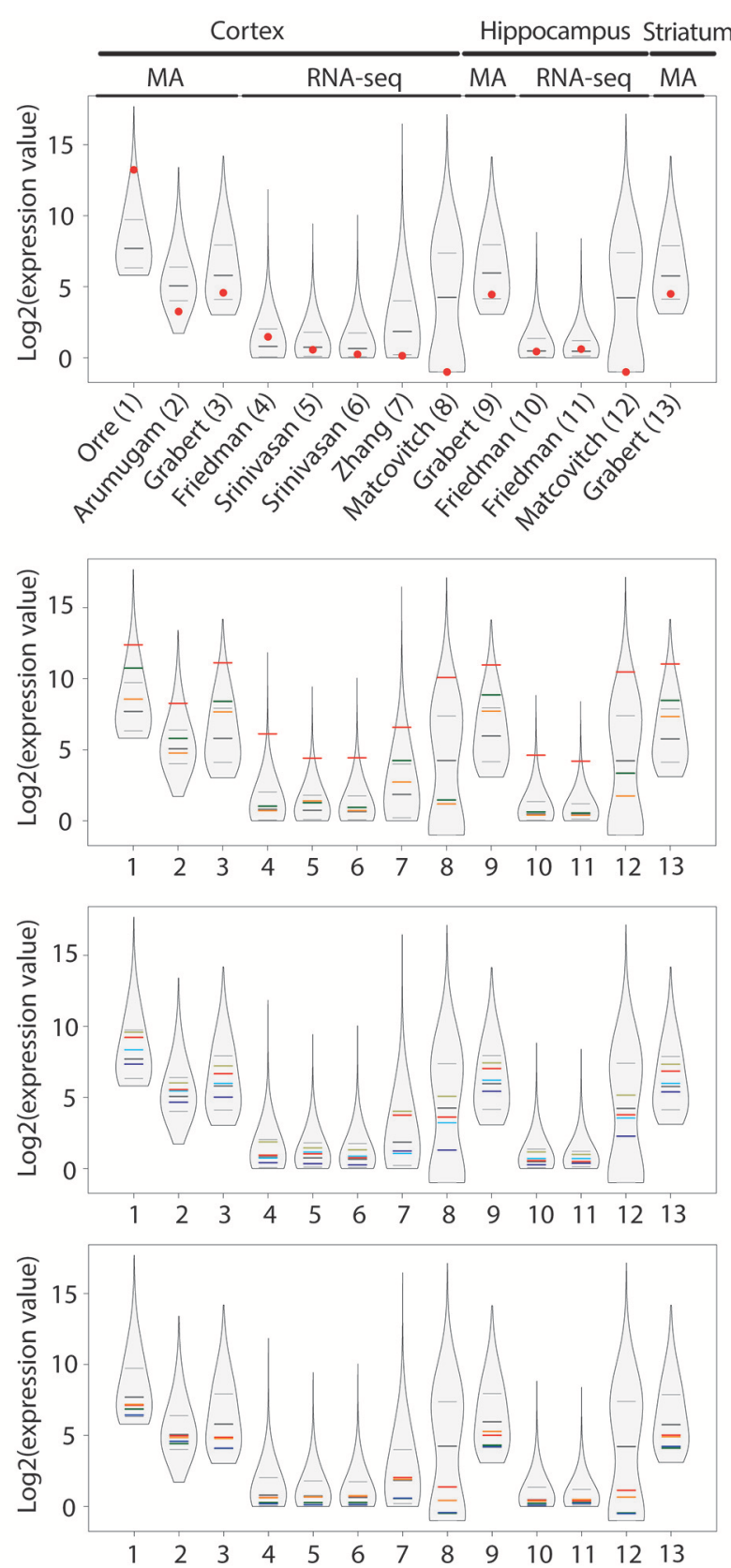

FIGURE 2 | Oprm1 and gene clusters expression in mouse cortex, hippocampus and striatum microglia datasets. Expression levels of the genes identified in each dataset are represented in violin plots demonstrating the median, as well as the 25th and 75th quartiles. Datasets are represented by name of the first author in (A) which are denoted numerically in (B-D). In mouse cortex and hippocampus, microglia were assayed with either microarrays (MA) or RNA-sequencing (RNA-seq) as underlined on (A) and assayed with MA only in the striatum (dataset \#13). (A) Oprm1 expression values were distributed below and above the median independent of the assay used to measure gene expression. RNA-seq datasets (\#8 and 12) yielded to no expression of Oprm1 in microglia. The highest Oprm1 expression value was yielded by dataset \#1 in cortical microglia. (B) Among the Myeloid gene clusters, the Microglia-related gene module was the mostly highly expressed in all datasets analyzed, with expression values distributed about the 75th quantile. (C) Proliferation-related genes were the most highly expressed within the Activation pattern clusters, distributed within the 75th quantile. (D) Neuron-, GABAergic-, and Astrocyte-related genes had expression values that were below the median in most of the datasets analyzed. In the cortex and hippocampus, the Excitatory neuron- and Astrocyte-related genes are distributed along the median for datasets \#5-7 and \#10, 11.

in $10 \%$ normal horse serum/0.1\% Triton X-100/PBS overnight at $4^{\circ} \mathrm{C}$. The sections were then washed 3 times in $0.1 \%$ Triton $\mathrm{X}$ 100/PBS and incubated with secondary antibodies and Hoechst for $1 \mathrm{~h}$ at room temperature, in the same solution as the primary antibodies. After washing 3 times in $0.1 \%$ Triton/PBS and once in PBS alone, slides were mounted with the S3023 aqueous 


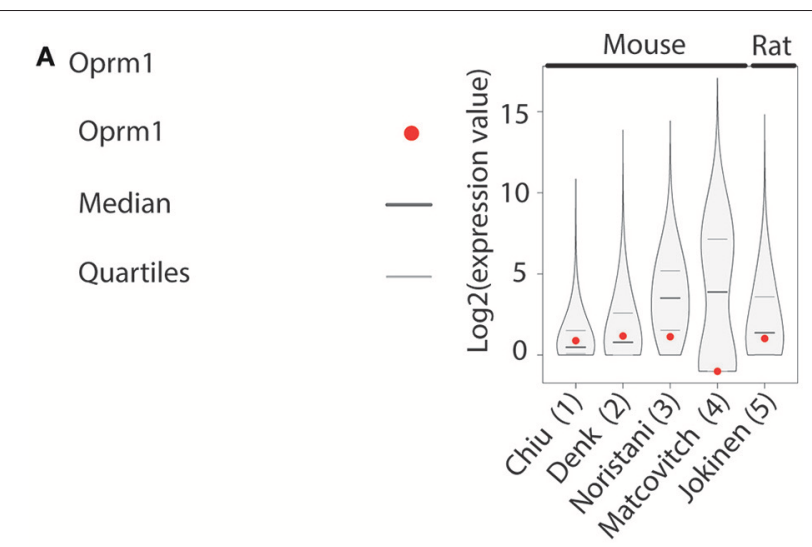

B Myeloid cells

Microglia

Macrophages

Neutrophil-monocyte
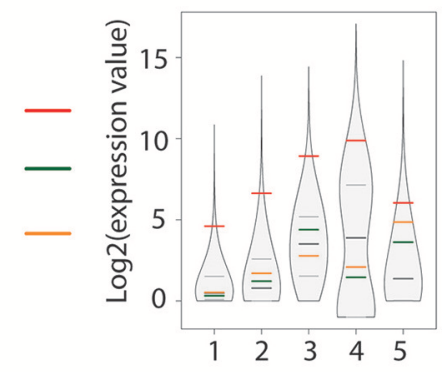

C Activation patterns

Interferon

Proliferation

LPS

Neurodegeneration
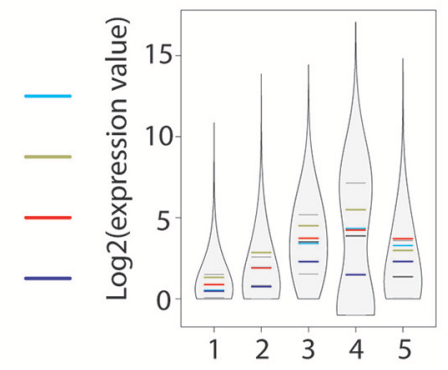

D Neurons and Astrocytes

Neurons

Excitatory

GABAergic

Astrocytes

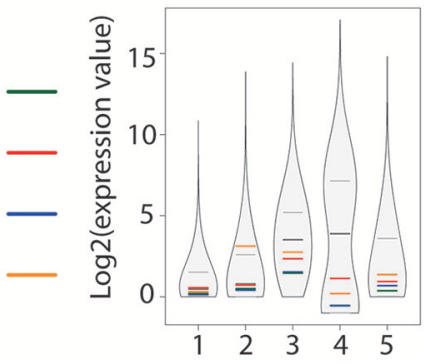

FIGURE 3 | Oprm1 and gene clusters expression in rodent spinal cord microglia datasets. Expression levels of the genes identified in each dataset are represented in violin plots demonstrating the median, as well as the 25th and 75 th quartiles. Datasets are represented by name of the first author in (A) which are denoted numerically in (B-D). Gene expression in the spinal cord was assayed with RNA-seq exclusively. (A) Oprm1 expression values ranged from above the median to undetected level (\#4). (B) In the Myeloid gene cluster, the Microglia- related genes were the mostly highly expressed in all datasets analyzed, with expression values distributed about the 75th quantile. (C) Proliferation-related genes were the most highly expressed within the Activation pattern clusters, except in dataset\#5, which had higher expression values for the LPS module in microglia purified from rat. Overall, the expression values of Activation patterns gene clusters were low compared to the Microglia-related genes. (D) Neuron-, GABAergic- and Astrocyte-related genes had expression values that were low compared to the Microglia-related genes except for dataset \#2 that show high Astrocyte-related module expression (B). mounting medium (DAKO). For staining cis-Golgi, trans-Glogi, and lysosomes, the rabbit polyclonal anti-GM130 antibody (11308-1-AP, Proteintech), the rabbit polyclonal anti-TGN38 antibody (NBP1-03495, Interchim), and the mouse monoclonal anti-Lamp1 antibody (H4A3, Developmental Studies Hybridoma Bank DSHB) were used. The optimal dilutions were set up in preliminary experiments. Anti-GM130, anti-TGN38, and antiLamp1 were used at dilutions 1:500, 1:200, and 1:200 (0.6, 5, $0.1 \mu \mathrm{g} / \mathrm{ml}$ ), respectively. The secondary antibodies used were the Cy5 conjugated donkey anti-mouse (1:1,000, 715-176-150, Jackson) and the Cy5 conjugated donkey anti-rabbit (1:500, 711-176-152, Jackson) Ig antibodies. Images were acquired with a confocal microscope (8UV, Leica Microsystem) using a 40x objective. The FIJI package for ImageJ software was used for image analysis (66). The percent of MOR-positive microglia among the CX3CR1-eGFP-positive microglia in the different brain and spinal cord regions were quantified on multiple microscopic fields from 3 female and 3 male adult animals. The number of fields counted and of cells examined are indicated in the Supplementary Table 5.

\section{Statistical Analysis}

Statistical analyses were performed with GraphPad Prism version 6.01 to investigate the correlations between Oprm1 expression and the different clusters mentioned above, as well as to compare MOR expression in CX3CR1-eGFP-positive microglia between male and female mice. Oprm1 and gene module $\mathrm{z}$-scores were all non-normally distributed and correlations were assessed with the Spearman's correlation test. The mean percentage of MORpositive and CX3CR1-eGFP-positive microglia were tested for normality as well and compared using the Unpaired Student's $t$-test or Mann Whitney test where applicable. The results are presented as the mean \pm SEM for microglia cell counts (percentages). A $p$-value of 0.05 or less was considered to be statistical significant.

\section{RESULTS}

\section{Oprm1/OPRM1 Expression in Rodent and Human Microglia Transcriptomics Datasets Microglia From Mouse Whole Brain}

Oprm1 was expressed in the twelve datasets analyzed (Figure 1A). Among those, six were issued from MA, see references (41-46) and six from RNA-seq, see references (47-52), as described in Supplementary Table 1. Oprm1 levels ranged from above the median $(n=4)$ to between the median and first quartile $(n=4)$ and below the first quartile $(n=4)$. Oprm1 expression tended to be more homogenous in MA than in RNA-seq datasets. The Myeloid gene modules defined by Friedman et al. (40) included Microglia, Macrophage, and Monocyte-neutrophil gene clusters. The Microglia gene cluster was highly expressed in all datasets, at the highest level of all gene clusters (Figure 1B). Macrophage and Neutrophil-monocyte cluster expression was distributed over and under the median among datasets (Figure 1B). The Proliferation cluster stood out as the most expressed among the Activation pattern gene sets, followed by the LPS, or Interferon and then Neurodegeneration 


\section{A Oprm1}

Oprm1

Median

Quartiles

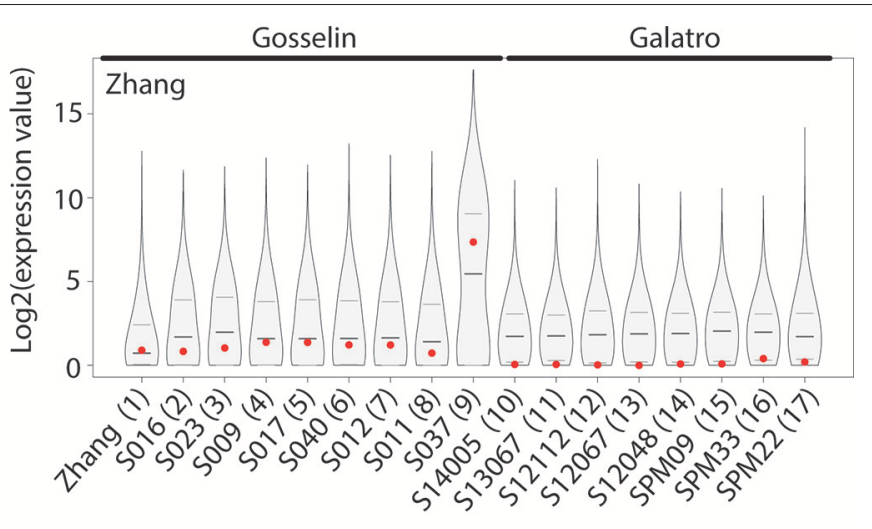

B Myeloid cells

Microglia

Macrophages

Neutrophil-monocyte

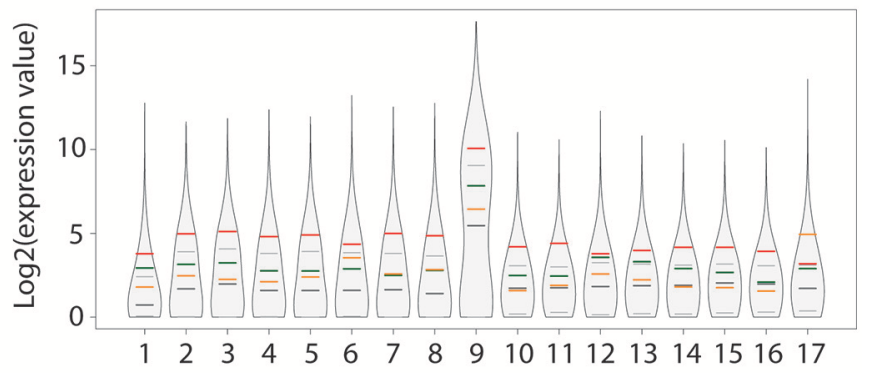

C Activation patterns

Interferon

Proliferation

LPS

Neurodegeneration

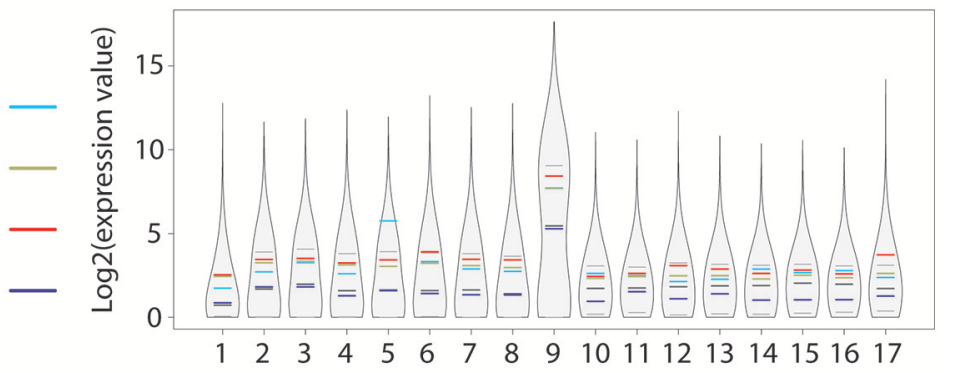

D Neurons and Astrocytes

Neurons

Excitatory

GABAergic

Astrocytes

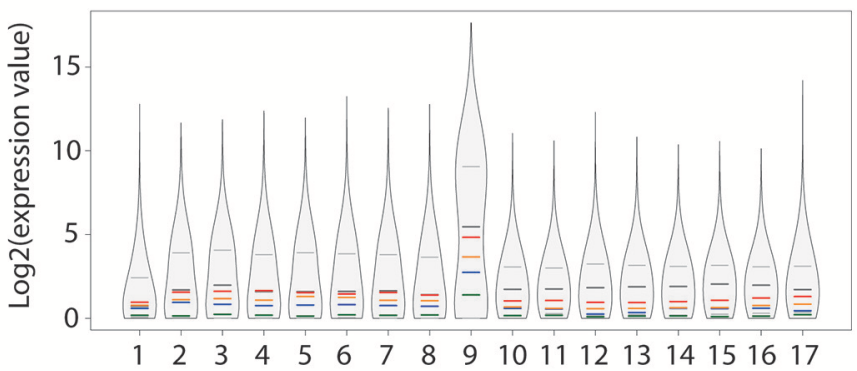

FIGURE 4 | Oprm1 and gene clusters expression in human cortex microglia datasets. Expression levels of the genes identified in each dataset are represented in violin plots demonstrating the median, as well as the 25th and 75th quartiles. In all datasets, microglia were assayed with RNA-seq. Gosselin datasets (\#2-9) comprised of microglia isolated from young males and females, while the other human datasets were from adult and aged postmortem samples of unknown gender (\#1) or exclusively males (10-17). Datasets are represented by name of the first author (A), which are denoted numerically in (B-D). (A) Oprm1 expression values were distributed below or along the median in the Zhang and Gosselin datasets (\#1 and 2-9) but consistently below the median in all the Galatro datasets. (B) In the Myeloid gene clusters, the Microglia- related genes were the most highly expressed in all datasets analyzed, with expression values distributed above the 75th quantile, except in dataset \#17 which had higher expression values for the Monocytes-Neutrophils-related genes. (C) Activation pattern gene clusters were highly expressed in purified human microglia, distributed within, and above the $75^{\text {th }}$ quantile. The majority of the datasets reported the highest expression values for the LPS-related genes. Interferon-related genes had the highest expression values in the datasets \#5, 10, 14, and 16 with causes of death associated with blood circulation and aneurysm. (D) Neuron-, GABAergic-, and Astrocyte-related genes had expression values along and below the median in dataset\#1 and those provided by Gosselin (\#2-9). Expression values were all below the median in the aged samples (\#10-17). 
gene sets (Figure 1C). The three neuronal (Neurons, Excitatory and GABAergic) gene sets were positioned below the median in nine of the datasets (Figure 1D). The Astrocyte cluster was below the median in eight of the datasets (Figure 1D).

\section{Microglia From Murine Cortex, Hippocampus, and Striatum}

In the cortex, Oprm1 expression was expressed in seven out of the eight datasets generated by either MA (53-55) or RNAseq $(40,56,57)$ and not detected in the dataset by MatcovitchNatan et al. derived from Massively parallel Single-cell RNAseq (Mars-seq) (58). Expression ranged from above the median in two datasets, between median, and first quartile in three datasets, below the first quartile in two datasets, to not being expressed in the Mars-seq dataset (Figure 2A). The dataset with very high Oprm1 expression was generated using the Agilent MA technology (53) while Affymetrix arrays were used in the other MA studies. In the hippocampus, Oprm1 was above the median in one dataset, ranged between the median, and first quartile in two datasets, but not expressed in the Mars-seq dataset. In the striatum dataset by Grabert et al., the Oprm1 was above the first quartile. The Microglia gene cluster was also highly expressed and the highest in all datasets (Figure 2B), similarly to whole mouse brain datasets. Macrophage and Neutrophilmonocyte module expression ranged among datasets from over to under the median, with the Macrophage cluster higher than that of Neutrophil-monocyte (Figure 2B). The Proliferation cluster stood out as the most expressed among the Activation pattern gene sets, followed by the LPS or Interferon and then Neurodegeneration gene sets (Figure 2C), comparably whole mouse brain data. The neuronal (Neurons, Excitatory and GABAergic) as well as the Astrocyte gene set were positioned below the median in nine of the datasets (Figure 2D).

\section{Rodent Spinal Cord}

The expression of Oprm 1 was analyzed in four mouse and one rat spinal microglia datasets obtained by RNA-seq (58-62). Oprm1 is expressed above the median in two mouse spinal cord datasets, below the first quartile in one data set, and not expressed in the Mars-seq dataset by Matcovitch-Natan et al. The rat spinal cord dataset showed Oprm1 level above the first quartile (Figure 3A). The Microglia gene cluster was also highly expressed in the five datasets (Figure 3B). Macrophage and Neutrophil-monocyte cluster expression varied among datasets from over to below the median (Figure 3B). In the Activation pattern gene modules, the Proliferation gene sets were the most expressed in mouse but not rat, whereas Neurodegeneration gene sets showed the lowest level (Figure 3C), similarly to what was found in mouse brain. The three neuronal (Neurons, Excitatory and GABAergic) gene sets were positioned below or at the median level while Astrocyte cluster expression differed across the datasets (Figure 3D).

\section{Human Cortex}

OPRM1 level was analyzed in microglia RNA-seq datasets generated from temporal cortex gray matter (63), different cortical areas (64), and parietal cortex (65) (Figure 4A). OPRM1 is expressed above the median in the dataset generated by Zhang et al. (63) for which microglia were purified from cortex gray matter collected on juvenile-adult individuals. In the study by Gosselin et al. (64) on cortical microglia enriched from young adults, OPRM1 levels ranged between the median and first quartile. In the $\mathrm{S} 037$ dataset issued from microglia collected from a brain tumor, OPRM1 was highly expressed. In the microglia datasets by Galatro et al. (65), collected from the parietal cortex of aged people (57-102 years), OPRM1 was around the first quartile and not expressed in the S12067 sample (Figure 4A). The Microglia gene module was the most expressed in all sets except one and the Macrophage cluster had generally a higher level than the Neutrophil-monocyte cluster (Figure 4B). Among the activation pattern gene sets, the LPS and Interferon sets showed mostly the highest levels, followed by the Proliferation cluster. The Neurodegeneration gene sets showed the lowest level of expression (Figure 4C). The values for the Excitatory and GABAergic clusters were higher than those of the Neurons cluster, which showed the lowest expression levels (Figure 4D), differing from mouse datasets. The Astrocyte cluster was below the median on most datasets (Figure 4D).

\section{Single Cell RNAseq Data Sets}

We investigated Oprm1 expression in microglia datasets from published single cell RNA-seq studies. Transcripts for Oprm1 were not found in the datasets which contained about 1,300 , $1,985,6,000,1,179,1,900-3,300$, and 1,169 and 800 genes expressed per cell, respectively (27, 67-72). Microglia datasets generated from microdissected basal ganglia nuclei led to the identification of 2,647 expressed genes per cell that did not include Oprm1 (73).

\section{Relationships Between Oprm1 Expression and Gene Clusters Myeloid Cells}

We investigated the relationships between Oprm1 expression and the expression of myeloid molecular signatures (modules) that are genes found to be co-expressed in the study by Friedman et al. (40). Microglia, in comparison with Macrophages cluster, had inverse trends regarding their correlations with Oprm1 in the rodent analyses (Figures 5-9). The Microglia module was positively correlated with Oprm1 (mouse cortex, mouse hippocampus, and rodent spinal cord), while the Macrophage cluster was negatively correlated with Oprm 1 .

In whole brain analysis, Oprm1 expression had a negative correlation with the Macrophage and Monocyte-neutrophil clusters, which was statistically significant with the Macrophage gene cluster only $(p=0.030)$. In the mouse cortex, there was a strong positive correlation between Oprm1 and the Microglia gene set which was statistically significant only for this cluster $(\mathrm{r}=0.955 ; p=0.0032)$ but not for the other myeloid sets (Figure 6). No significant correlation was found between the Monocyte-neutrophil gene cluster and any dataset (Figures 5-8).

\section{Activation Patterns}

We investigated the relationships between the expression of Oprm1 and of the myeloid activation modules as described (40). There was a negative correlation between Oprm1 and the LPS 
Myeloid cells
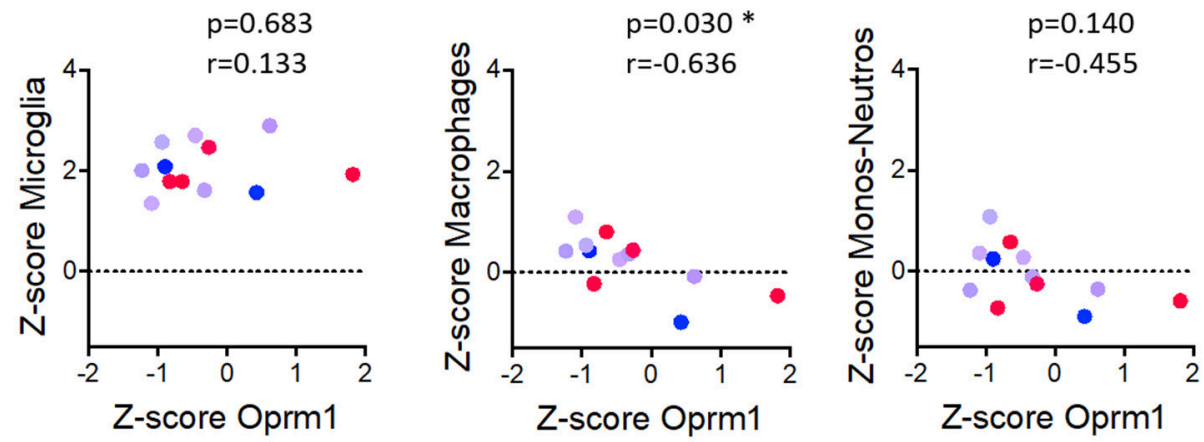

\section{Activation patterns}
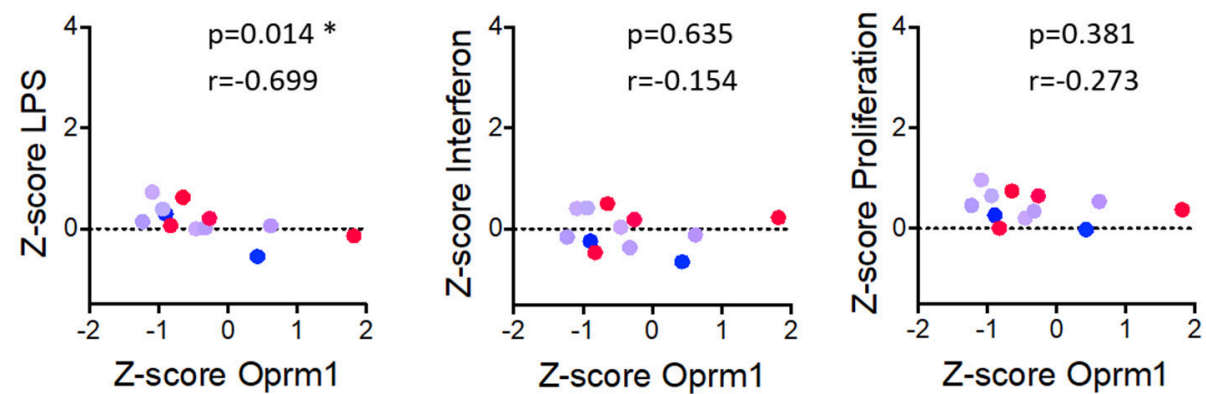

Neurons and Astrocytes
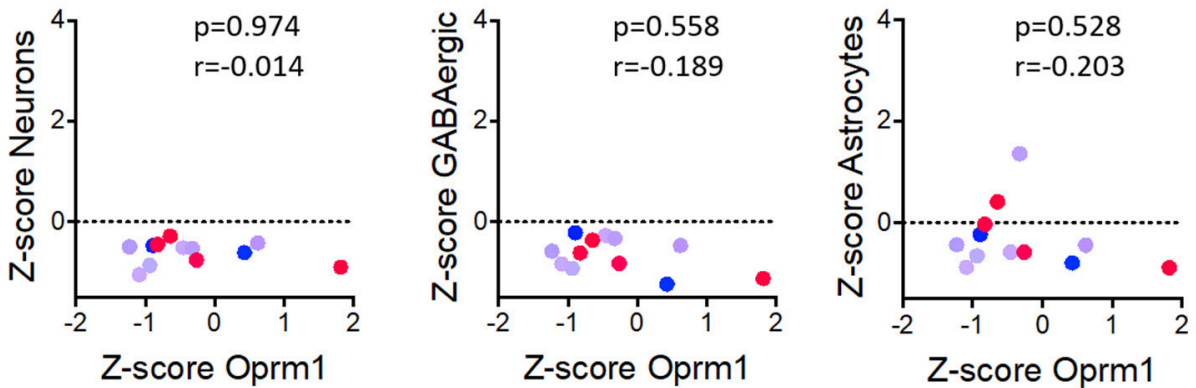
datasets. Oprm1 expression does not correlate with the microglia-related and the Monocyte-Neutrophil-related genes ( $p>0.05$, ns) but is significantly negatively correlated with the Macrophages-related genes ( $r=0.636$; $\left.{ }^{\star} p=0.03\right)$. Oprm1 expression has a negative correlation $(r<0)$ with all the Activation patterns-, Neurons, and Astrocytes gene clusters, which is only statistically significant for the LPS-related gene module $\left.{ }^{*} p=0.014\right)$. All correlations were determined with the Spearman's correlation test. 
Myeloid cells
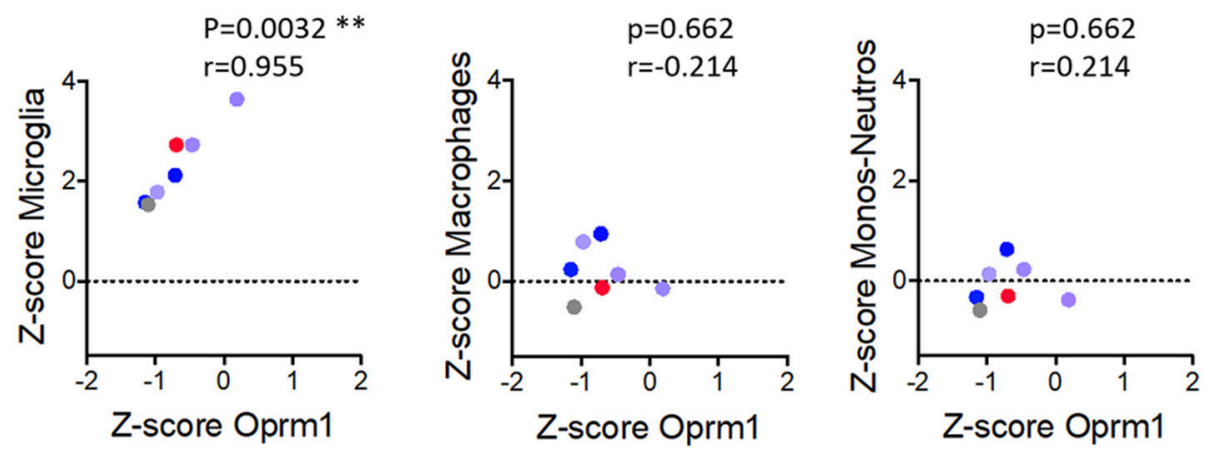

\section{Activation patterns}
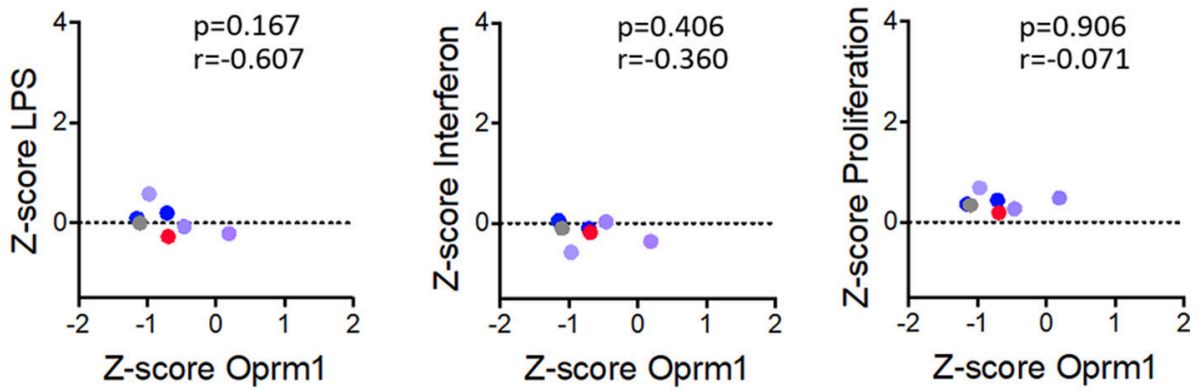

\section{Neurons and Astrocytes}
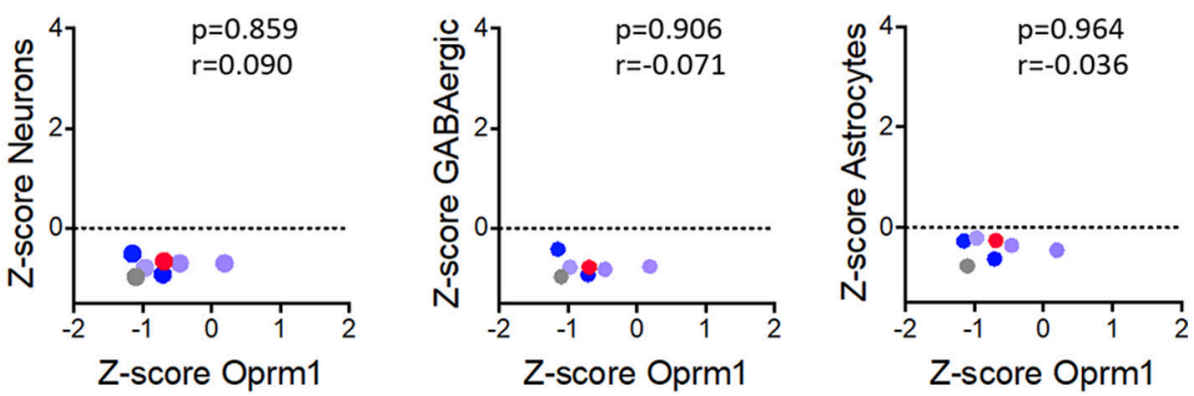

\section{- Female \\ - Male \\ Mixed \\ Non-available}

FIGURE 6 | Correlation of Oprm1 gene expression within the Myeloid cells, Activation patterns, and Neurons and Astrocytes gene clusters in mouse cortex datasets. Oprm1 expression is positively and significantly correlated with the microglia-related genes $\left(r=0.955\right.$; $\left.{ }^{* *} p=0.0032\right)$ but does not correlate with the Macrophagesand Monocyte-Neutrophils-related genes $(r \leq 0.214 ; p>0.05, \mathrm{~ns})$. Oprm1 expression does not correlate with any of the Activation patterns- $(r<0$; $p>0.05$, ns), Neurons or Astrocytes gene clusters ( $r \leq 0.09$; $p>0.05$, ns). All correlations were determined with the Spearman's correlation test. 


\section{Myeloid cells}

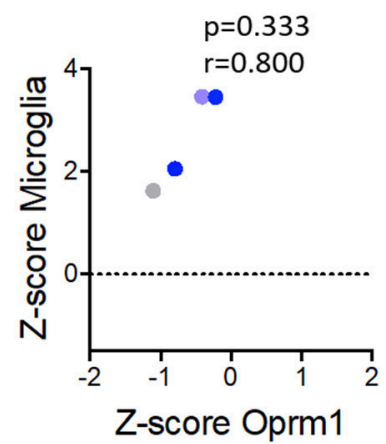

\section{Activation patterns}

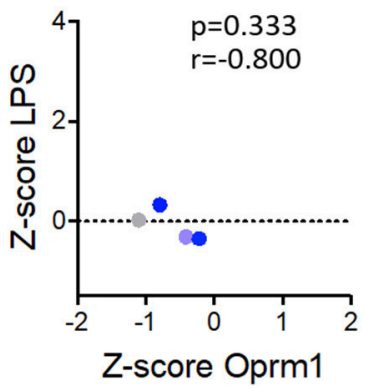

Neurons and Astrocytes

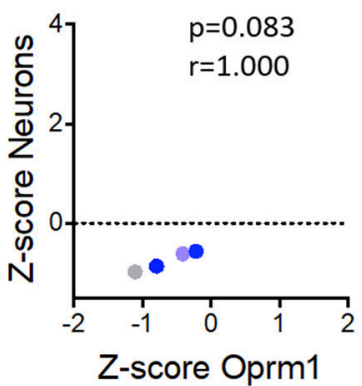

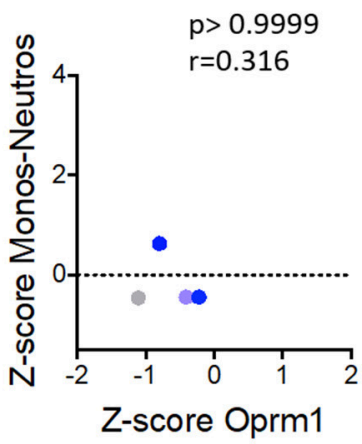

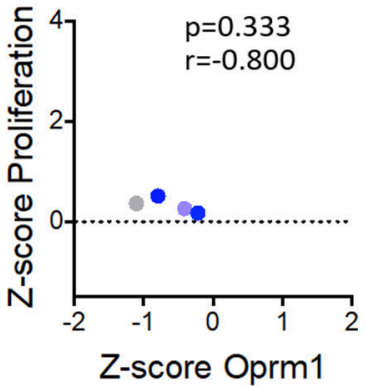

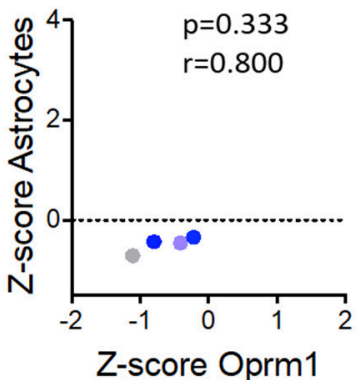

Male

Mixed

Non-available

FIGURE 7 | Correlation of Oprm1 gene expression within the Myeloid cells, Activation patterns and Neurons and Astrocytes gene clusters in mouse hippocampus datasets. There is no correlation between Oprm1 expression and all the gene clusters investigated in the mouse hippocampus datasets ( $p>0.05$, ns). Although Oprm1 expression seems to have a strongly positive correlation with the Neurons and Astrocytes gene clusters ( $r=1$ and $r=0.800$, respectively) this is not statistically significant $(p>0.05, n s)$. All correlations were determined with the Spearman's correlation test. 
Myeloid cells
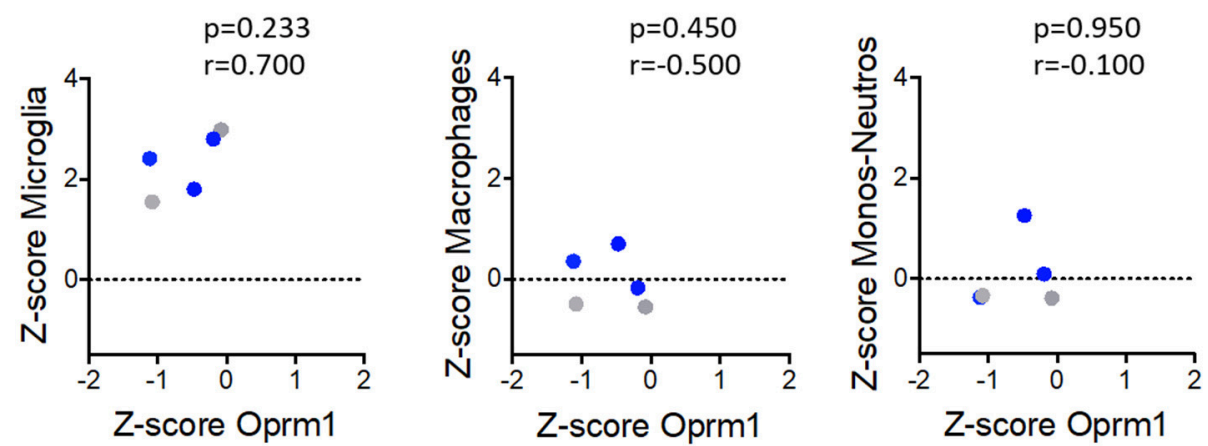

\section{Activation patterns}
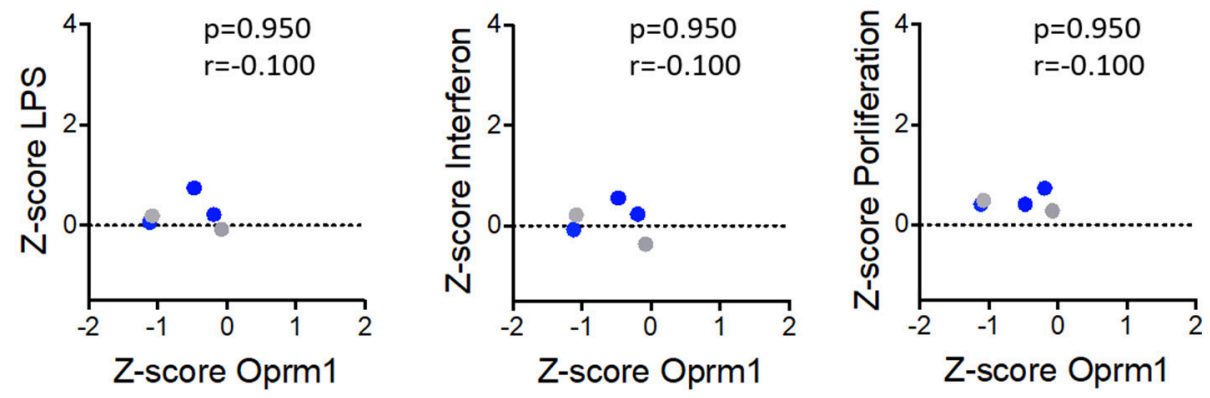

\section{Neurons and Astrocytes}
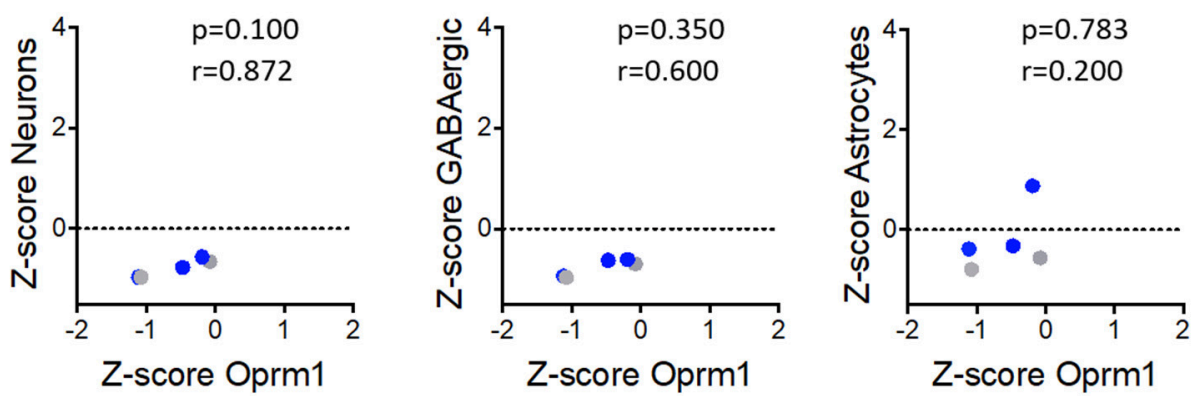

- Male

Mixed

Non-available

FIGURE 8 | Correlation of Oprm1 gene expression within the Myeloid cells, Activation patterns, and Neurons and Astrocytes gene clusters in rodent spinal cord datasets. Oprm1 expression has a positive correlation $(r=0.700)$ with the Microglia-related genes but which is not statistically significant $(p>0.05$, ns). Oprm1 expression is negatively correlated $(r<0)$ with Macrophages, Monocytes-Neutrophils- related genes, and Activation patterns gene clusters, but this is not statistically significant $(r<0 ; p>0.05, \mathrm{~ns})$. Oprm1 expression has a positive correlation with the Neurons $(r>0.5)$ which is not statistically significant $(p>0.05$, ns). There is no correlation between Oprm1 expression and Astrocytes gene clusters ( $r=0.2 ; p=0.783$, ns). All correlations were determined with the Spearman's correlation test. 


\section{Myeloid cells}

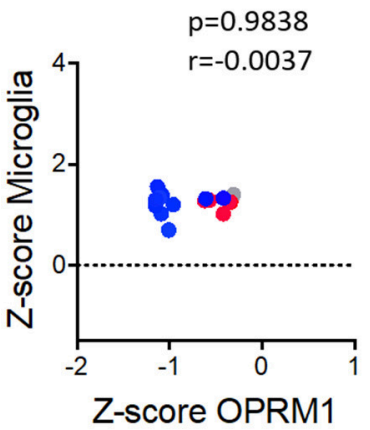

Activation patterns

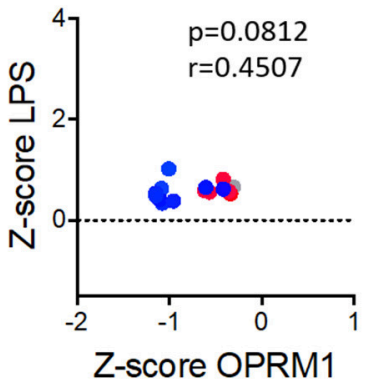

Neurons and Astrocytes
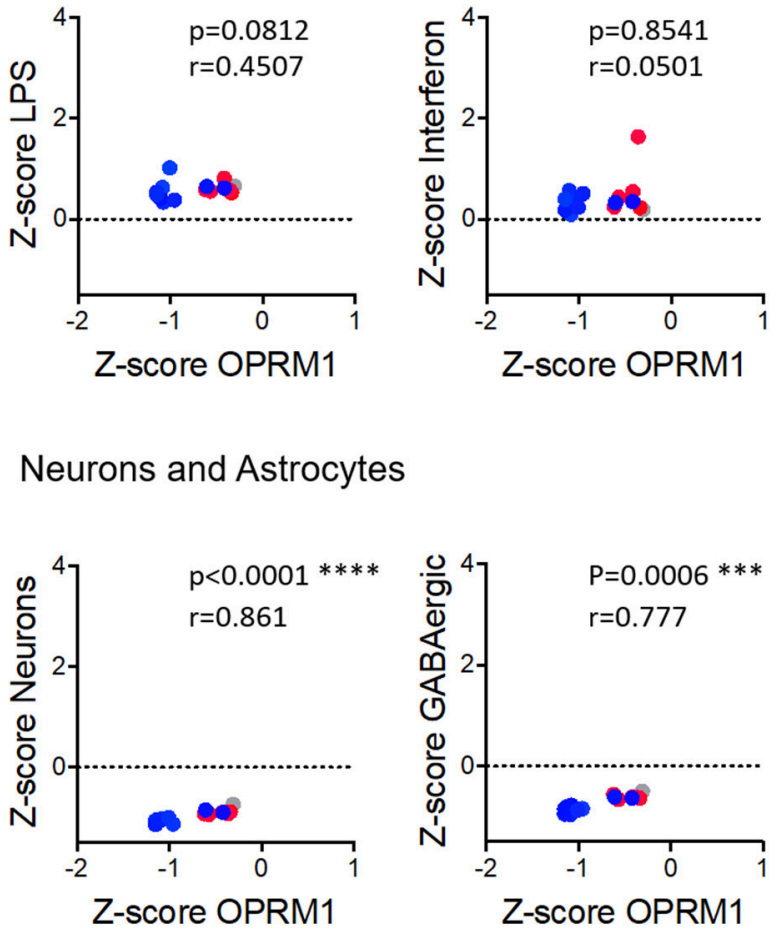
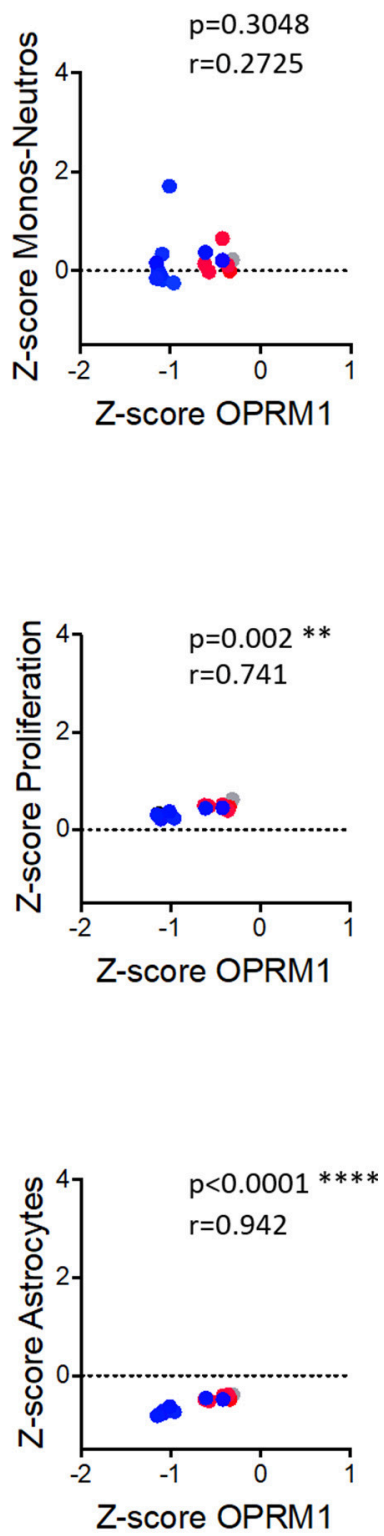

- Female

- Male

Non-available

FIGURE 9 | Correlation of Oprm1 gene expression within the Myeloid cells, Activation patterns and Neurons and Astrocytes gene clusters in human cortex datasets. Oprm1 expression had a negative correlation with Microglia- and Macrophage-related genes $(r<0)$ and a positive correlation with the Monocytes-Neutrophils-related genes which were not statistically significant $(p>0.05$; ns). Within the Activation patterns-related genes, Oprm 1 expression has a weak positive correlation $(r<0.5)$ which is not statistically significant for the LPS-related genes $(p>0.5)$. There is a statistically significant positive correlation between Oprm1 expression and the Proliferation-related genes $\left(r=0.741 ;{ }^{* *} p=0.002\right)$. There is a statistically significant positive correlation between Oprm1 expression and the GABAergic- $(r>0.5$; $\left.{ }^{* * *} p=0.0006\right)$, Neurons-, and Astrocytes-related genes $\left(r>0.5 ;{ }^{\star * \star *} p<0.0001\right)$. 
gene cluster in the mouse whole brain datasets (Figure 5) and a positive correlation between OPRM1 and the Proliferationrelated gene level for the human cortex datatsets (Figure 9). No correlation was found between Oprm1 level and the Interferon cluster in any of the dataset studied (Figures 5-9).

\section{Neurons and Astrocytes}

The analysis for relationships between Oprm1 expression and expression of the Neuron and GABAergic neuronal signatures indicated no correlation between Oprm 1 and these modules for whole mouse brain, cortex, hippocampus, and rodent spinal cord datasets (Figures 5-8). The $p$ and $r$ values obtained from the analysis of hippocampus and spinal cord data suggest that more datasets are required to increase the power of the correlation analysis. For the human cortex datasets, a positive correlation was found between Oprm1 expression and expression of the Neuronal and Astrocyte gene modules (Figure 9).

\section{MOR Expression in CX3cr1-eGFP Microglia MOR Expression in Brain and Spinal Cord}

The Figures 10, 11 show that Cx3cr1-eGFP-positive cells display the typical microglia morphology with no neuronal, astrocytic or lymphocytic shapes, confirming previous findings on colocalization of CX3CR1-eGFP with the CD11b or Iba1 microglia and macrophage markers $(37,74,75)$ but not neuronal, astrocytic, or oligodendrocytic markers $(76,77)$ in the brain. MOR is expressed in microglia of different brain regions in female and male $\mathrm{Cx} 3 \mathrm{cr} 1$-eGFP-MOR-mCherry mice, in the Frontal Cortex (FCx), Nucleus Accumbens (NAcc), Central Amygdala (CeA), Ventral Tegmental Area (VTA), and Periaqueductal Gray (PAG) (Figure 10). The percent of microglia expressing MOR-mCherry protein ranges from 35.4 \pm 4.1 to $51.6 \pm 3.5$ (Supplementary Table 5 ). The percentage of MOR-positive microglia was calculated in the FCx, NAcc, CeA, and PAG of female and male brains. The VTA showed a lower percentage of microglia containing MOR in females than in males which was statistically significant $(p=0.0037)$ (Supplementary Table 5).

In spinal cord dorsal horn, $\mathrm{MOR}$ is expressed in microglia of both female and male Cx3cr1-eGFP-MORmCherry mice (Figures 11A,B, Supplementary Video 1, Supplementary Table 5). The percentage of microglia expressing MOR ranged from $37.1 \pm 2.7$ to $44.542 .4 \pm 2.6$ in cervical, thoracic and lumbar segments, with no statistical difference between the segments (Supplementary Table 5). There were no statistically significant differences in the percentages of MOR-expressing microglia between males and females with 36.9 $\pm 2.3 \%$ and $36 \pm 2.4 \%, 38.8 \pm 2.1 \%$ and $42.4 \pm 2.5 \%$, and 37.1 $\pm 2.7 \%$ and $39.5 \pm 2.3 \%$ in cervical, thoracic, and lumbar spinal segments, respectively (Supplementary Table 5).

\section{MOR-Golgi-lysosome Localization}

To specifically localize MOR within the intracellular compartments of spinal microglia, we labeled Cis-Golgi, trans-Golgi, and lysosomes with GM130, TGN38, and Lamp1 antibodies, respectively. Microglial MOR co-localized with GM-130 (Figure 11C) and TGN38 (Figure 11D) but not with Lamp1 (Figure 11E). Together, this indicates that microglial MOR is localized in cis and trans-Golgi rather than lysosomes.

\section{DISCUSSION}

Altogether the transcriptomics and imaging data show MOR expression in microglia of all analyzed mammalian brain areas and the spinal cord. The expression of Oprm1 transcripts and MOR protein in microglia was shown previously by using RT-qPCR or antibody based techniques, respectively (914, 16). Although two papers found no expression of MOR in microglia by using the same above-mentioned techniques $(21,22)$, a link between microglial activation and MOR expression and function was demonstrated, mostly in in vitro studies on murine primary microglia cultures. Low concentrations of morphine and DAMGO (a selective MOR agonist) activated rat microglia and this activation was blocked by the MOR-selective antagonist D-Phe-Cys-Tyr-D-Trp-ArgThr-Pen-Thr-NH2 (CTAP) (15). Morphine increased Toll-like Receptors (TLRs) expression which was attenuated by the MORselective antagonist D-Phe-Cys-Tyr-D-Trp-Orn-Thr-Pen-Thr$\mathrm{NH} 2$ (CTOP). Additionally, this morphine effect was abolished in cultured microglia of MOR knockout (KO) mice (78). In murine microglia, morphine increased the expression of cytokines and activated the PKCe-Akt-ERK1/2 kinase pathway and these effects were inhibited by Oprm1 RNA silencing as well as by CTAP $(14,16)$. A low dose of morphine enhanced NF-kB activity via MOR that was blocked by CTAP while a morphine high dose triggered a TLR4-mediated non-opioid response (18). One study has shown that, in vivo, the morphine-induced increase in microglial P2X4R ATP-receptor was blocked by (-)-naloxone but not by its $(+)$-naloxone enantiomer, implicating microglial MOR rather than TLR4 in OIH (17). MOR implication in $\mathrm{OIH}$ has been confirmed at the genetic level with the use of global MOR KO mice $(21,79)$. Regarding the implication of TLR4 in $\mathrm{OIH}$, the study of TLR4 KO mice has led to controversial results as two papers showed that OIH was abolished in TLR4 KO mice while two others showed that it was maintained $(6,7)$. Moreover, TLR4-independent activation of NF-kB by morphine was shown together with increased Tumor Necrosis Factor alpha $(\mathrm{TNF} \alpha)$, suggesting $\mathrm{TNF} \alpha$ signaling as a novel pathway for morphine action on microglia (80). However, whether this effect of morphine on microglia activation occurs directly through the microglial MOR or indirectly via a neuronal MOR still remains to be determined and could be addressed in upcoming studies such as with conditional MOR-KO targeted at microglia or other cell types.

The detection of Oprm1/OPRM1 in 27 out of 30 rodent datasets and 16 out of 17 human whole genome transcriptomics datasets issued from microglia ex vivo validates the presence of MOR transcripts in microglia. From this analysis, we could conclude that in the mammalian central nervous system, microglia of the whole brain, cerebral cortex, hippocampus, and spinal cord all contain Oprm1/OPRM1 messenger. Singlecell RNA-seq currently allows detection of only a few thousand 


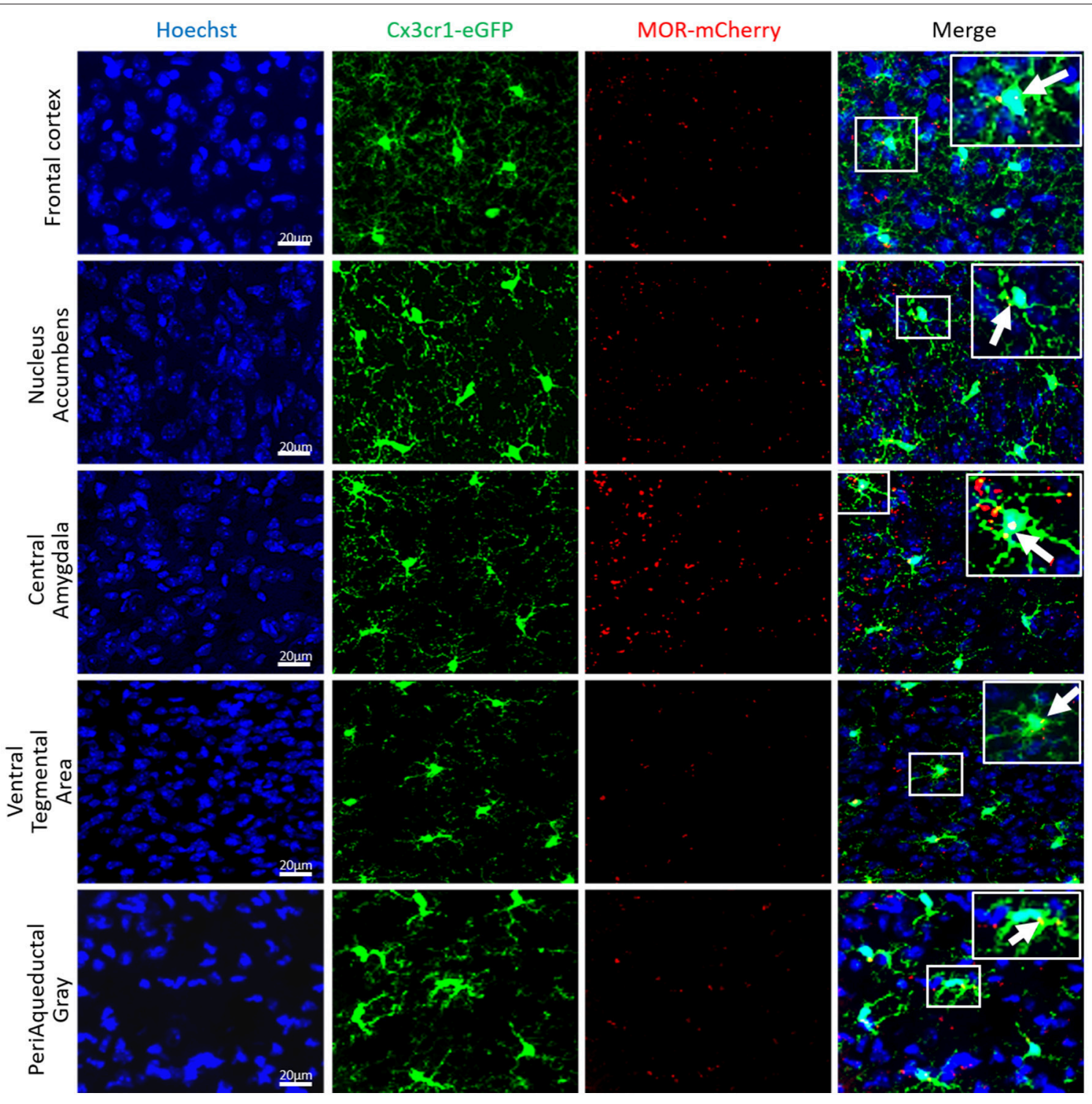

FIGURE 10 | MOR is expressed in mouse brain microglia. Photomicrographs from coronal sections of Frontal cortex (FCX), Nucleus Accumbens (NAcc), Central Amygdala (CeA), Ventral Tegmental Area (NTA), and Periaqueductal Gray (PAG) of Cx3Cr1-eGFP-MOR-mCherry mice show the colocalization (orange) of CX3CR1-eGFP (green), and MOR-mCherry (red) denoted by white arrows. Scale bar $=20 \mu \mathrm{m}$.

of the most highly expressed transcripts within a specimen. Therefore, this approach is used for determining differential gene expression among the highly expressed genes and cannot analyze complete transcriptomes (81). As Oprm1 is overall expressed around or below the median within the reported transcriptomes, the transcripts would not be contained among the few thousands of highly expressed genes detected by single-cell RNA-seq studies. This lack of expression is found also in the Mars-seq datasets (58).

The analysis of the relationships between expression of Oprm 1 and of the different gene clusters led to interesting insights. First, Figures 5-9 show that the z-scores for Microglia-related gene expression are globally above 1 while $\mathrm{z}$-scores for neurons or astrocytes were below 0 , indicating a significant enrichment in microglia compared to other cell types. The positive correlation observed between Oprm 1 and the Microglia module in mouse cortex further supports the presence of Oprm1 expression in microglia. Such a correlation was not found for the mouse whole brain or spinal cord datasets, possibly reflecting the diverse cellular heterogeneity in these tissues compared to a defined region like the cortex. Indeed, the whole brain comprises many distinct regions, and microglia from other brain regions implicated in opioid response or pain, such as VTA, NAcc, or PAG, should be further profiled at the molecular level in order to characterize the global expression of microglial Oprm1. Neurons, GABAergic neurons and Astrocytes gene clusters correlate with OPRM1 in the human cortex datasets but not in datasets 
A

Male

Hoechst/Cx3Cr1-eGFP/

MOR-mCherry
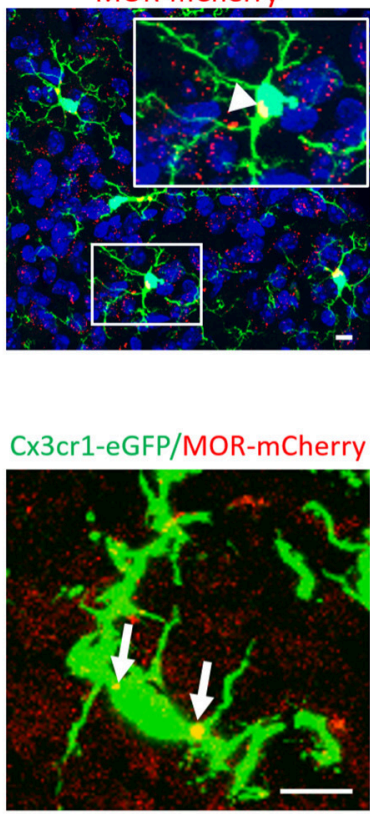

D Cx3cr1-eGFP/MOR-mCherry

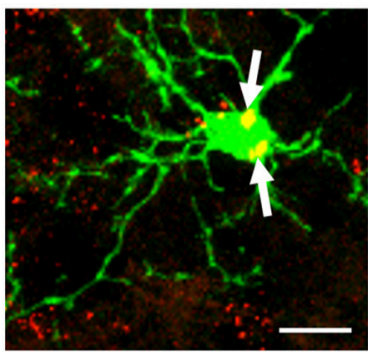

E Cx3cr1-eGFP/MOR-mCherry

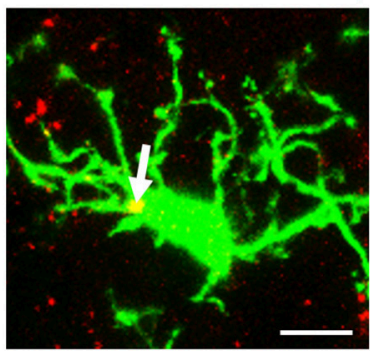

B

\section{Female}

Hoechst/Cx3Cr1-eGFP/

MOR-mCherry

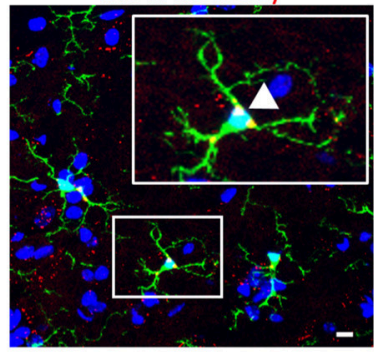

GM130

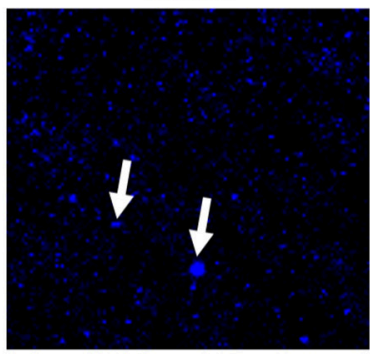

TGN38

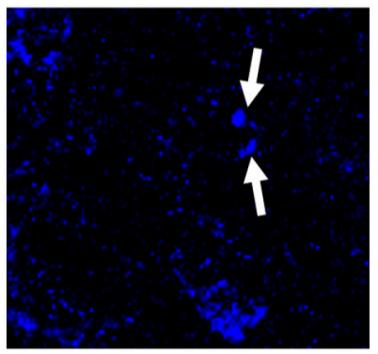

Lamp1

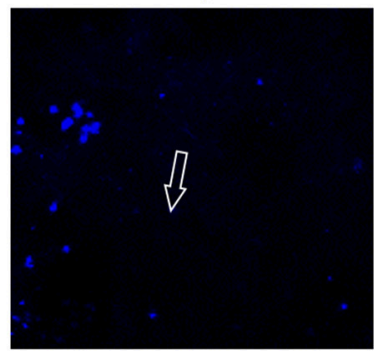

Merge

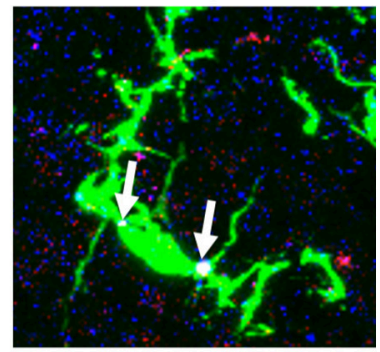

Merge

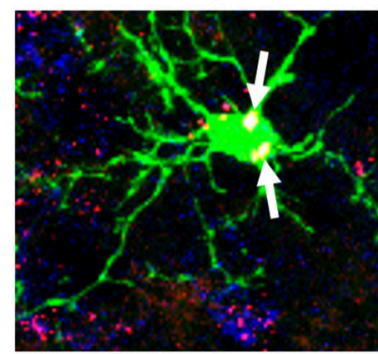

Merge

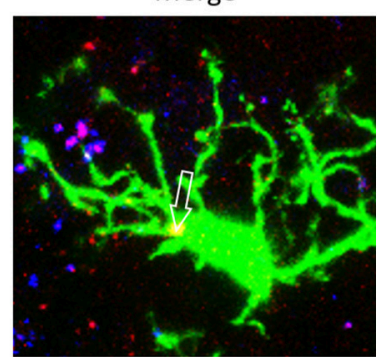

FIGURE 11 | MOR is expressed in microglia of the mouse spinal cord. (A,B) Photomicrographs from transverse sections of the lumbar spinal cord, at the level of the dorsal horn, demonstrate a colocalization (orange) of MOR-mCherry (red) with CX3CR1-eGFP-positive microglia (green) in adult male (A) and female (B) Cx3Cr1-eGFP-MOR-mCherry mice, indicated by white arrow heads. Scale bar $=10 \mu \mathrm{m}$. (C-E) High magnification photomicrographs of CX3CR1-eGFP positive microglia (green) that colocalize (orange) with MOR-mCherry (red) are indicated with white arrows. Scale bar $=10 \mu \mathrm{m}$. (C) Anti-GM130 labeling (blue) colocalizes (pink-white) with MOR-mCherry in the cis-Golgi of CX3CR1-eGFP positive microglia, indicated with white arrows. (D) Anti-TGN38 labeling (blue) colocalizes (pink-white) with MOR-mCherry in the trans-Golgi of CX3CR1-eGFP positive microglia, indicated with white arrows. (E) Anti-Lamp1 labeling (blue) of lysosomes does not colocalize with MOR-mCherry in CX3CR1-eGFP positive microglia, indicated with open arrows. The white (closed) arrow indicate colocalization of CX3CR1-eGFP with MOR-mCherry (orange) alone.

from rodents. A significantly higher content of neuronal and astrocyte gene transcripts in FACS-isolated microglia compared to cultured microglia has already been reported, and was suggested to result from synapse-related mRNAs that were phagocytosed by the microglia (19). The same phenomenon may contribute to the positive correlation found between OPRM1 
and Neurons and Astrocytes modules in the human datasets, despite their very low expression levels in the datasets. In addition to the transcriptomic analysis, imaging of the Cx3cr1-eGFPMOR-mCherry reporter line confirmed MOR expression by microglia of the FCx, NAcc, CeA, VTA, PAG, and spinal cord. Furthermore, co-localization of microglial MOR with cis- and trans-Golgi compartments but not lysosomes strongly supports the protein synthesis and post-translational processing of MOR within microglial cells.

Importantly, as discussed by Richard M Ransohoff, microglia polarization into M1 and M2 subtypes appears too simplistic and does not reflect the diversity of microglia phenotypes or profiles induced by internal and external environmental factors that include central nervous system region, sex, age, and health state revealed by high-throughput analyses (82). Some reports on microglia transcriptomics have defined co-regulated gene subsets or modules to allow for classification of microglia into subtypes expressing high levels of specific gene modules. We analyzed the 47 microglia datasets and established three main activation module profiles related to Interferon, LPS and Proliferation, in relation to Oprm1 expression. We found a lack of, or a poor correlation between Orpm1 and these modules. There is a negative correlation between Oprm1 and the LPS-related module in whole mouse brain datasets, and a positive correlation between OPRM1 and the Proliferation-related module in human cortex datasets. These low correlations may be explained by the use of datasets from naive, non-stimulated rodents microglia in which the LPS-related, Interferon-related, and Proliferation related genes are expressed at the basal low level.

Several lines of evidence support a functional impact of MOR on microglia activity. Morphine increased spinal microglial p38 and extracellular receptor kinase (ERK) phosphorylation, leading to an augmented microglial production of proinflammatory cytokines, and other pronociceptive molecules as well as their receptors and hence to increased pain (15). Morphine also enhances the activity of microglial ATP-gated P2X7R and P2X4R receptors in the spinal cord, leading to increased Brain-derived neurotrophic factor (BDNF) release from these cells. This in turn downregulates $\mathrm{K}-\mathrm{Cl}$ co-transporter $\mathrm{KCC} 2$ expression in GABAergic neurons enhancing their excitability and thus increased pain $(8,17,20,83)$. Other spinal microglial-mediated responses for morphine analgesic tolerance and hyperalgesia involve large conductance $\mathrm{Ca} 2+$-activated $\mathrm{K}+$ channels (84) and genes of the DPA12/TREM2 and potassium intermediate/small conductance calcium activated channels KCNN4 pathways (62). Furthermore, bidirectional cross-talks between chemokine receptors and opioid receptors mediate opioid analgesic tolerance (85). Also, microglial pannexin-1 channels attenuate morphine withdrawal in rodents but are not involved in opioid analgesic tolerance, thus identifying pannexin-1 as a novel mediator of specific morphine actions in microglia $(86,87)$. Peripheral nerve injury leads to microglial activation in several brain structures involved in pain sensation or emotion including the VTA and amygdala (88). Within the VTA, chronic pain, chronic opiate treatment, and opioid withdrawal induce a dysregulation of BDNF in microglia that impacts on GABAergic neurons and disrupts the dopaminergic pathway leading to defects in reward
$(89,90)$. Therefore, specific regulations in microglia appear to mediate opioid reward and link chronic pain and opioid dependence $(91,92)$ that may be mediated by MOR activity within different subsets of microglia.

With the use of the Cx3cr1-eGFP-MOR-mCherry reporter mouse, we have shown that, overall, $35-51 \%$ microglia express MOR, suggesting that the receptor may be expressed by a particular microglial subset and that only MOR-expressing microglia will respond to opioid treatment. Future studies are necessary to address how MOR expression within different microglial subsets may impact on their functionality, under physiological, and pathological conditions. The specific ablation of the Oprm1 gene in the different subtypes of resting or activated microglia by targeted Cre/Lox technology would require the identification of unique and very selective gene markers for each of these subtypes and the generation of the corresponding Cre mouse lines. In the same line, optogenetic approaches allow the evaluation of opioid signaling and behavior elicited by specific spatiotemporal patterns of MOR activation in neurons (93) that could be applied to microglia. Another aspect of MOR activation is ligand-biased signaling where different ligands stimulate differential cell responses $(94,95)$. We report an intracellular localization of MOR within the Golgi apparatus of microglia that suggests a local production of MOR in these cells. MOR function in microglia could be investigated by novel biosensors that have been assayed on transfected cell lines and primary neuronal cultures, revealing a cell localization bias for opioid receptor activation by endogenous, and exogenous opioids (96).

As sex differences are an important factor for microglial contribution to pain $(35,36)$, we investigated MOR expression in microglia from adult females and males. The transcriptomic datasets did not contain enough numbers per sex for a full determination of sex effect, see Figures 5-9. The analysis of brain sections from the Cx3cr1-eGFP-MOR-mCherry mice indicated a comparable ratio of microglia containing MOR in female and male animals in different brain and spinal regions, except in the VTA where significantly lower percentages of microglia expressed MOR in females than males. It would therefore be interesting to determine whether microglial MOR would influence the regulatory mechanisms in the dopaminergic system in females as has been previously described for males in the VTA $(89,90)$. Also, sex differences in microglia activity were found in the PAG where a significantly higher microglial activation profile was found in females compared to males (97). In the spinal cord, the microglial P2X4R-mediated hyperalgesia showed sexual dimorphism (98). Altogether this suggests that transcriptomic profiles of microglia in chronic pain models or following chronic drug administration may help to identify Oprm1-linked mechanisms associated with alterations in microglial functions. However, sex differences may exert a weaker influence on morphine-induced analgesic tolerance and hyperalgesia than on other forms of chronic pain like neuropathic pain. Indeed, these morphine adverse effects occurred similarly in male and female mice (79) and no clear impact was shown on OIH (6).

The question of the factors influencing the opioid effects on microglia physiology should be further explored in the future by taking into account the genetic diversity, sex differences, and 
pathological states (26). The importance of genetic background on opioid tolerance, hyperalgesia (6), and misuse (99) has been well-established in both clinical and preclinical settings. The rodent datasets analyzed in the present study were from mouse of C57BL/6 genetic background except four datasets issued from other strains, genetic background, or species. In the future, the study of Oprm1 expression may be broadened to a larger number of rodent strains to get a more comprehensive view. In addition, all human microglia datasets were collected from cortical areas and there is a need for studies on microglia from spinal cord and other brain regions involved in pain and addiction. The correlations between rodent and human transcriptomes may be studied as well. Galatro et al. have shown an overlap between the two species, with similar microglia core genes expressed by both human and mouse microglia including the main markers CX3CR1, P2YR12, and Itgam. There were also dissimilarities due to differences in environment and medical condition as mouse microglia were isolated from healthy mice while human donors suffered from pathologies that led to their death, potentially influencing microglial genes regulation, and expression (65).

Neuroinflammatory mechanisms underlie opioid-induced pain sensitization $(6,7)$ comprising of reciprocal signaling between neurons and neuroinflammatory cells including microglia (100). OIH, together with abuse liability, contribute to the opioid crisis that is epidemic in North America and becoming prevalent in Europe as well (101). Therefore, the elucidation of mechanisms involved in opioid deleterious effects would help to develop new strategies for safer analgesic clinical intervention. Altogether, this work on microglial Oprm1 expression and previous studies open the way to the exploration of the role of microglial MOR in response to opiates, in particular to analgesic tolerance, $\mathrm{OIH}$, and physical dependence elicited by their chronic use.

\section{DATA AVAILABILITY STATEMENT}

Data are available on request.

\section{REFERENCES}

1. Gaveriaux-Ruff C. Opiate-induced analgesia: contributions from mu, delta and kappa opioid receptors mouse mutants. Curr Pharm Des. (2013) 19:7373-81. doi: 10.2174/138161281942140105163727

2. Maldonado R, Banos JE, Cabanero D. Usefulness of knockout mice to clarify the role of the opioid system in chronic pain. Br J Pharmacol. (2018) 175:2791-808. doi: 10.1111/bph.14088

3. Siuda ER, Carr R, III, Rominger DH, Violin JD. Biased mu-opioid receptor ligands: a promising new generation of pain therapeutics. Curr Opin Pharmacol. (2017) 32:77-84. doi: 10.1016/j.coph.2016.11.007

4. Gunther T, Dasgupta P, Mann A, Miess E, Kliewer A, Fritzwanker S, et al. Targeting multiple opioid receptors - improved analgesics with reduced side effects? Br J Pharmacol. (2018) 175:2857-68. doi: 10.1111/bph.13809

5. Ji RR, Chamessian A, Zhang YQ. Pain regulation by non-neuronal cells and inflammation. Science (2016) 354:572-7. doi: 10.1126/science.aaf8924

6. Roeckel LA, Le Coz GM, Gaveriaux-Ruff C, Simonin F. Opioid-induced hyperalgesia: Cellular and molecular mechanisms. Neuroscience (2016) 338:160-82. doi: 10.1016/j.neuroscience.2016.06.029

\section{AUTHOR CONTRIBUTIONS}

TM analyzed data and contributed to the writing of the manuscript. EA, DD, NM, and CG-R designed experiments, analyzed data and contributed to the writing of the manuscript. DR performed experiments. DM provided the MOR-mCherry mouse line and contributed to the writing of the manuscript.

\section{FUNDING}

This work has received funding from the European Union Seventh Framework programme (FP7-Health-2013-Innovation) under grant agreement 1602919 (CG-R), by Université de Strasbourg (CG-R); by Frame program Investissements d'Avenir ANR-10-IDEX-0002-02 ANR-10-LABX-0030-INRT (CG-R). DM was supported by CNRS.

\section{ACKNOWLEDGMENTS}

We thank Ivan Weinsanto for critical review of the manuscript. We thank Marco Antonio Mendoza Parra for his advice and help on R software, Yann Herault and Maria del Mar Muniz Moreno and Marc Ruff for discussions and Sandra Bour for assistance with the figures. We thank Annie-Paule Sibler, Bruno Kieffer, Odile Lecompte, Yves Nominé, and Bruno Chatton for their kind help. We thank the ICS Animal facility, Isabelle Goncalves, Alexis Simon, Caroline Pham and Maira Fontaine for animal care. We thank Pascal Kessler at IGBMC, Elvire Guiot at the MCI and all members of the imaging facility for advice on imaging. We thank Fabien Alpy and Ioannis Manolaras for the anti-golgi and anti-Lamp1 antibodies.

\section{SUPPLEMENTARY MATERIAL}

The Supplementary Material for this article can be found online at: https://www.frontiersin.org/articles/10.3389/fpsyt. 2018.00726/full\#supplementary-material

7. Rivat C, Ballantyne J. The dark side of opioids in pain management: basic science explains clinical observation. Pain Rep. (2016) 1:e570. doi: 10.1097/PR9.0000000000000570

8. Leduc-Pessah H, Weilinger NL, Fan CY, Burma NE, Thompson RJ, Trang T. Site-specific regulation of P2X7 receptor function in microglia gates morphine analgesic tolerance. J Neurosci. (2017) 37:10154-72. doi: 10.1523/JNEUROSCI.0852-17.2017

9. Chao CC, Hu S, Shark KB, Sheng WS, Gekker G, Peterson PK. Activation of mu opioid receptors inhibits microglial cell chemotaxis. J Pharmacol Exp Ther. (1997) 281:998-1004.

10. Calvo CF, Cesselin F, Gelman M, Glowinski J. Identification of an opioid peptide secreted by rat embryonic mixed brain cells as a promoter of macrophage migration. Eur J Neurosci. (2000) 12:2676-84. doi: 10.1046/j.1460-9568.2000.00145.x

11. Turchan-Cholewo J, Dimayuga FO, Ding Q, Keller JN, Hauser KF, Knapp PE, et al. Cell-specific actions of HIV-Tat and morphine on opioid receptor expression in glia. J Neurosci Res. (2008) 86:2100-10. doi: 10.1002/jnr.21653

12. Mika J, Popiolek-Barczyk K, Rojewska E, Makuch W, Starowicz K, Przewlocka B. Delta-opioid receptor analgesia is independent of microglial 
activation in a rat model of neuropathic pain. PLoS ONE (2014) 9:e104420. doi: 10.1371/journal.pone. 0104420

13. Popiolek-Barczyk K, Piotrowska A, Makuch W, Mika J. Biphalin, a dimeric enkephalin, alleviates LPS-induced activation in rat primary microglial cultures in opioid receptor-dependent and receptor-independent manners. Neural Plast. (2017) 2017:3829472. doi: 10.1155/2017/3829472

14. Merighi S, Gessi S, Varani K, Fazzi D, Stefanelli A, Borea PA. Morphine mediates a proinflammatory phenotype via mu-opioid receptorPKCvarepsilon-Akt-ERK1/2 signaling pathway in activated microglial cells. Biochem Pharmacol. (2013) 86:487-96. doi: 10.1016/j.bcp.2013.05.027

15. Horvath RJ, DeLeo JA. Morphine enhances microglial migration through modulation of P2X4 receptor signaling. J Neurosci. (2009) 29:998-1005. doi: 10.1523/JNEUROSCI.4595-08.2009

16. Merighi S, Gessi S, Varani K, Fazzi D, Mirandola P, Borea PA. Cannabinoid $\mathrm{CB}(2)$ receptor attenuates morphine-induced inflammatory responses in activated microglial cells. Br J Pharmacol. (2012) 166:2371-85. doi: 10.1111/j.1476-5381.2012.01948.x

17. Ferrini F, Trang T, Mattioli TA, Laffray S, Del'Guidice T, Lorenzo LE, et al. Morphine hyperalgesia gated through microglia-mediated disruption of neuronal Cl(-) homeostasis. Nat Neurosci. (2013) 16:183-92. doi: $10.1038 / \mathrm{nn} .3295$

18. Gessi S, Borea PA, Bencivenni S, Fazzi D, Varani K, Merighi S. The activation of mu-opioid receptor potentiates LPS-induced NF-kB promoting an inflammatory phenotype in microglia. FEBS Lett. (2016) 590:2813-26. doi: $10.1002 / 1873-3468.12313$

19. Beutner C, Linnartz-Gerlach B, Schmidt SV, Beyer M, Mallmann MR, Staratschek-Jox A, et al. Unique transcriptome signature of mouse microglia. Glia (2013) 61:1429-42. doi: 10.1002/glia.22524

20. Horvath RJ, Romero-Sandoval EA, De Leo JA. Inhibition of microglial $\mathrm{P} 2 \mathrm{X} 4$ receptors attenuates morphine tolerance, Iba1, GFAP and mu opioid receptor protein expression while enhancing perivascular microglial ED2. Pain (2010) 150:401-13. doi: 10.1016/j.pain.2010.02.042

21. Corder G, Tawfik VL, Wang D, Sypek EI, Low SA, Dickinson JR, et al. Loss of mu opioid receptor signaling in nociceptors, but not microglia, abrogates morphine tolerance without disrupting analgesia. Nat Med. (2017) 23:164-73. doi: $10.1038 / \mathrm{nm} .4262$

22. Kao SC, Zhao X, Lee CY, Atianjoh FE, Gauda EB, Yaster M, et al. Absence of mu opioid receptor mRNA expression in astrocytes and microglia of rat spinal cord. Neuroreport (2012) 23:378-84. doi: 10.1097/WNR.0b013e3283522e1b

23. Denk F. Don't let useful data go to waste. Nature (2017) 543:7. doi: $10.1038 / 543007 \mathrm{a}$

24. Sousa C, Biber K, Michelucci A. Cellular and molecular characterization of microglia: a unique immune cell population. Front Immunol. (2017) 8:198. doi: $10.3389 /$ fimmu.2017.00198

25. Crotti A, Ransohoff RM. Microglial physiology and pathophysiology: insights from genome-wide transcriptional profiling. Immunity (2016) 44:505-15. doi: 10.1016/j.immuni.2016.02.013

26. Hirbec H, Noristani HN, Perrin FE. Microglia responses in acute and chronic neurological diseases: what microglia-specific transcriptomic studies taught (and did Not Teach) us. Front Aging Neurosci. (2017) 9:227. doi: 10.3389/fnagi.2017.00227

27. Avey D, Sankararaman S, Yim AKY, Barve R, Milbrandt J, RDM. Single-Cell RNA-Seq uncovers a robust transcriptional response to morphine by glia. Cell Rep. (2018) 24:3619-29. doi: 10.1016/j.celrep.2018.08.080

28. Prinz M, Erny D, Hagemeyer N. Ontogeny and homeostasis of CNS myeloid cells. Nat Immunol. (2017) 18:385-92. doi: 10.1038/ni.3703

29. Yona S, Kim KW, Wolf Y, Mildner A, Varol D, Breker M, et al. Fate mapping reveals origins and dynamics of monocytes and tissue macrophages under homeostasis. Immunity (2013) 38:79-91. doi: 10.1016/j.immuni.2012.12.001

30. Jung S, Aliberti J, Graemmel P, Sunshine MJ, Kreutzberg GW, Sher A, et al. Analysis of fractalkine receptor CX(3)CR1 function by targeted deletion and green fluorescent protein reporter gene insertion. Mol Cell Biol. (2000) 20:4106-14. doi: 10.1128/MCB.20.11.4106-4114.2000

31. Erbs E, Faget L, Scherrer G, Matifas A, Filliol D, Vonesch JL, et al. A mu-delta opioid receptor brain atlas reveals neuronal cooccurrence in subcortical networks. Brain Struct Funct. (2015) 220:677-702. doi: $10.1007 /$ s00429-014-0717-9
32. Mogil JS. Sex differences in pain and pain inhibition: multiple explanations of a controversial phenomenon. Nat Rev Neurosci. (2012) 13:859-66. doi: $10.1038 / \mathrm{nrn} 3360$

33. Sorge RE, Mapplebeck JC, Rosen S, Beggs S, Taves S, Alexander JK, et al. Different immune cells mediate mechanical pain hypersensitivity in male and female mice. Nat Neurosci. (2015) 18:1081-3. doi: 10.1038/nn.4053

34. Taves S, Berta T, Liu DL, Gan S, Chen G, Kim YH, et al. Spinal inhibition of p38 MAP kinase reduces inflammatory and neuropathic pain in male but not female mice: Sex-dependent microglial signaling in the spinal cord. Brain Behav Immun. (2016) 55:70-81. doi: 10.1016/j.bbi.2015.10.006

35. Sorge RE, Totsch SK. Sex differences in pain. J Neurosci Res. (2017) 95:127181. doi: 10.1002/jnr.23841

36. Mapplebeck JC, Beggs S, Salter MW. Molecules in pain and sex: a developing story. Mol Brain (2017) 10:9. doi: 10.1186/s13041-017-0289-8

37. Kim I, Mlsna LM, Yoon S, Le B, Yu S, Xu D, et al. A postnatal peak in microglial development in the mouse hippocampus is correlated with heightened sensitivity to seizure triggers. Brain Behav. (2015) 5:e00403. doi: $10.1002 /$ brb3.403

38. Voronova A, Yuzwa SA, Wang BS, Zahr S, Syal C, Wang J, et al. Migrating interneurons secrete fractalkine to promote oligodendrocyte formation in the developing mammalian brain. Neuron (2017) 94:500-16.e9.

39. Tay TL, Savage JC, Hui CW, Bisht K, Tremblay ME. Microglia across the lifespan: from origin to function in brain development, plasticity and cognition. J Physiol. (2017) 595:1929-45. doi: 10.1113/JP272134

40. Friedman BA, Srinivasan K, Ayalon G, Meilandt WJ, Lin H, Huntley MA, et al. Diverse brain myeloid expression profiles reveal distinct microglial activation states and aspects of alzheimer's disease not evident in mouse models. Cell Rep. (2018) 22:832-47. doi: 10.1016/j.celrep.2017.12.066

41. Wang Y, Cella M, Mallinson K, Ulrich JD, Young KL, Robinette ML, et al. TREM2 lipid sensing sustains the microglial response in an Alzheimer's disease model. Cell (2015) 160:1061-71. doi: 10.1016/j.cell.2015.01.049

42. Verheijden S, Beckers L, Casazza A, Butovsky O, Mazzone M, Baes M. Identification of a chronic non-neurodegenerative microglia activation state in a mouse model of peroxisomal beta-oxidation deficiency. Glia (2015) 63:1606-20. doi: 10.1002/glia.22831

43. Poliani PL, Wang Y, Fontana E, Robinette ML, Yamanishi Y, Gilfillan S, et al. TREM2 sustains microglial expansion during aging and response to demyelination. J Clin Invest. (2015) 125:2161-70. doi: 10.1172/JCI77983

44. Erny D, Hrabe de Angelis AL, Jaitin D, Wieghofer P, Staszewski O, David E, et al. Host microbiota constantly control maturation and function of microglia in the CNS. Nat Neurosci. (2015) 18:965-77. doi: 10.1038/nn.4030

45. Szulzewsky F, Pelz A, Feng X, Synowitz M, Markovic D, Langmann T, et al. Glioma-associated microglia/macrophages display an expression profile different from M1 and M2 polarization and highly express Gpnmb and Spp1. PLoS ONE (2015) 10:e0116644. doi: 10.1371/journal.pone.0116644

46. Pyonteck SM, Akkari L, Schuhmacher AJ, Bowman RL, Sevenich L, Quail DF, et al. CSF-1R inhibition alters macrophage polarization and blocks glioma progression. Nat Med. (2013) 19:1264-72. doi: 10.1038/nm.3337

47. Bruttger J, Karram K, Wortge S, Regen T, Marini F, Hoppmann N, et al. Genetic cell ablation reveals clusters of local self-renewing microglia in the mammalian central nervous system. Immunity (2015) 43:92-106. doi: 10.1016/j.immuni.2015.06.012

48. Lavin Y, Winter D, Blecher-Gonen R, David E, Keren-Shaul H, Merad $M$, et al. Tissue-resident macrophage enhancer landscapes are shaped by the local microenvironment. Cell (2014) 159:1312-26. doi: 10.1016/j.cell.2014.11.018

49. Bennett ML, Bennett FC, Liddelow SA, Ajami B, Zamanian JL, Fernhoff $\mathrm{NB}$, et al. New tools for studying microglia in the mouse and human CNS. Proc Natl Acad Sci USA (2016) 113:E1738-46. doi: 10.1073/pnas. 15255 28113

50. Gosselin D, Link VM, Romanoski CE, Fonseca GJ, Eichenfield DZ, Spann NJ, et al. Environment drives selection and function of enhancers controlling tissue-specific macrophage identities. Cell (2014) 159:1327-40. doi: 10.1016/j.cell.2014.11.023

51. Krasemann S, Madore C, Cialic R, Baufeld C, Calcagno N, El Fatimy R, et al. The TREM2-APOE pathway drives the transcriptional phenotype of dysfunctional microglia in neurodegenerative diseases. Immunity (2017) 47:566-81.e9. doi: 10.1016/j.immuni.2017.0 
52. Zhao D, Mokhtari R, Pedrosa E, Birnbaum R, Zheng D, Lachman HM. Transcriptome analysis of microglia in a mouse model of Rett syndrome: differential expression of genes associated with microglia/macrophage activation and cellular stress. Mol Autism. (2017) 8:17. doi: 10.1186/s13229-017-0134-z

53. Orre M, Kamphuis W, Osborn LM, Melief J, Kooijman L, Huitinga I, et al. Acute isolation and transcriptome characterization of cortical astrocytes and microglia from young and aged mice. Neurobiol Aging. (2014) 35:1-14. doi: 10.1016/j.neurobiolaging.2013.07.008

54. Arumugam TV, Manzanero S, Furtado M, Biggins PJ, Hsieh YH, Gelderblom $\mathrm{M}$, et al. An atypical role for the myeloid receptor Mincle in central nervous system injury. J Cereb Blood Flow Metab. (2017) 37:2098-111. doi: 10.1177/0271678X16661201

55. Grabert K, Michoel T, Karavolos MH, Clohisey S, Baillie JK, Stevens MP, et al. Microglial brain region-dependent diversity and selective regional sensitivities to aging. Nat Neurosci. (2016) 19:504-16. doi: 10.1038/nn.4222

56. Srinivasan K, Friedman BA, Larson JL, Lauffer BE, Goldstein LD, Appling LL, et al. Untangling the brain's neuroinflammatory and neurodegenerative transcriptional responses. Nat Commun. (2016) 7:11295. doi: 10.1038/ncomms11295

57. Zhang Y, Chen K, Sloan SA, Bennett ML, Scholze AR, O'Keeffe S, et al. An RNA-sequencing transcriptome and splicing database of glia, neurons, and vascular cells of the cerebral cortex. J Neurosci. (2014) 34:11929-47. doi: 10.1523/JNEUROSCI.1860-14.2014

58. Matcovitch-Natan O, Winter DR, Giladi A, Vargas Aguilar S, Spinrad A, Sarrazin S, et al. Microglia development follows a stepwise program to regulate brain homeostasis. Science (2016) 353:aad8670. doi: $10.1126 /$ science.aad 8670

59. Chiu IM, Morimoto ET, Goodarzi H, Liao JT, O’Keeffe S, Phatnani HP, et al. A neurodegeneration-specific gene-expression signature of acutely isolated microglia from an amyotrophic lateral sclerosis mouse model. Cell Rep. (2013) 4:385-401. doi: 10.1016/j.celrep.2013.06.018

60. Denk F, Crow M, Didangelos A, Lopes DM, McMahon SB. Persistent alterations in microglial enhancers in a model of chronic pain. Cell Rep. (2016) 15:1771-81. doi: 10.1016/j.celrep.2016.04.063

61. Noristani HN, Gerber YN, Sabourin JC, Le Corre M, Lonjon N, MestreFrances N, et al. RNA-Seq analysis of microglia reveals time-dependent activation of specific genetic programs following spinal cord injury. Front Mol Neurosci. (2017) 10:90. doi: 10.3389/fnmol.2017.00090

62. Jokinen V, Sidorova Y, Viisanen $H$, Suleymanova I, Tiilikainen $H$, Li Z, et al. Differential Spinal and supraspinal activation of glia in a rat model of morphine tolerance. Neuroscience (2018) 375:10-24. doi: 10.1016/j.neuroscience.2018.01.048

63. Zhang Y, Sloan SA, Clarke LE, Caneda C, Plaza CA, Blumenthal PD, et al. Purification and characterization of progenitor and mature human astrocytes reveals transcriptional and functional differences with mouse. Neuron (2016) 89:37-53. doi: 10.1016/j.neuron.2015.11.013

64. Gosselin D, Skola D, Coufal NG, Holtman IR, Schlachetzki JCM, Sajti E, et al. An environment-dependent transcriptional network specifies human microglia identity. Science (2017) 356:eaal3222. doi: 10.1126/science.aal3222

65. Galatro TF, Holtman IR, Lerario AM, Vainchtein ID, Brouwer N, Sola PR, et al. Transcriptomic analysis of purified human cortical microglia reveals age-associated changes. Nat Neurosci. (2017) 20:1162-71. doi: $10.1038 / \mathrm{nn} .4597$

66. Schindelin J, Arganda-Carreras I, Frise E, Kaynig V, Longair M, Pietzsch T, et al. Fiji: an open-source platform for biological-image analysis. Nat Methods (2012) 9:676-82. doi: 10.1038/nmeth.2019

67. Darmanis S, Sloan SA, Zhang Y, Enge M, Caneda C, Shuer LM, et al. A survey of human brain transcriptome diversity at the single cell level. Proc Natl Acad Sci USA (2015) 112:7285-90. doi: 10.1073/pnas.15071 25112

68. Zeisel A, Munoz-Manchado AB, Codeluppi S, Lonnerberg P, La Manno $\mathrm{G}$, Jureus A, et al. Brain structure. Cell types in the mouse cortex and hippocampus revealed by single-cell RNA-seq. Science (2015) 347:1138-42. doi: $10.1126 /$ science.aaa 1934

69. Tasic B, Menon V, Nguyen TN, Kim TK, Jarsky T, Yao Z, et al. Adult mouse cortical cell taxonomy revealed by single cell transcriptomics. Nat Neurosci. (2016) 19:335-46. doi: 10.1038/nn.4216
70. Keren-Shaul H, Spinrad A, Weiner A, Matcovitch-Natan O, DvirSzternfeld R, Ulland TK, et al. A unique microglia type associated with restricting development of alzheimer's disease. Cell (2017) 169:1276-90.e17. doi: 10.1016/j.cell.2017.05.018

71. Mathys H, Adaikkan C, Gao F, Young JZ, Manet E, Hemberg M, et al. Temporal tracking of microglia activation in neurodegeneration at single-cell resolution. Cell Rep. (2017) 21:366-80. doi: 10.1016/j.celrep.2017.09.039

72. Saunders AME, Wysoker A, Goldman M, Krienen F, Bien E, Baum M, et al. Molecular diversity and specializations among the cells of the adult mouse brain. Cell (2018) 174:1015-30. doi: 10.1016/j.cell.2018.07.028

73. De Biase LM, Schuebel KE, Fusfeld ZH, Jair K, Hawes IA, Cimbro R, et al. Local cues establish and maintain region-specific phenotypes of basal ganglia microglia. Neuron (2017) 95:341-56.e6. doi: 10.1016/j.neuron.2017.06.020

74. Squarzoni P, Oller G, Hoeffel G, Pont-Lezica L, Rostaing P, Low D, et al. Microglia modulate wiring of the embryonic forebrain. Cell Rep. (2014) 8:1271-9. doi: 10.1016/j.celrep.2014.07.042

75. Kälin S, Miller KR, Kälin RE, Jendrach M, Witzel C, FL H. CNS myeloid cells critically regulate heat hyperalgesia. J Clin Invest. (2018) 128:2774-86. doi: 10.1172/JCI95305

76. Cardona AE, Pioro EP, Sasse ME, Kostenko V, Cardona SM, Dijkstra IM, et al. Control of microglial neurotoxicity by the fractalkine receptor. Nat Neurosci. (2006) 9:917-24. doi: 10.1038/nn1715

77. Parkhurst CN, Yang G, Ninan I, Savas JN, Yates JR III, Lafaille $\mathrm{JJ}$, et al. Microglia promote learning-dependent synapse formation through brain-derived neurotrophic factor. Cell (2013) 155:1596-609. doi: 10.1016/j.cell.2013.11.030

78. Dutta R, Krishnan A, Meng J, Das S, Ma J, Banerjee S, et al. Morphine modulation of toll-like receptors in microglial cells potentiates neuropathogenesis in a HIV-1 model of coinfection with pneumococcal pneumoniae. J Neurosci. (2012) 32:9917-30. doi: 10.1523/JNEUROSCI.0870-12.2012

79. Roeckel LA, Utard V, Reiss D, Mouheiche J, Maurin H, Robe A, et al. Morphine-induced hyperalgesia involves mu opioid receptors and the metabolite morphine-3-glucuronide. Sci Rep. (2017) 7:10406. doi: 10.1038/s41598-017-11120-4

80. Xie N, Gomes FP, Deora V, Gregory K, Vithanage T, Nassar ZD, et al. Activation of mu-opioid receptor and Toll-like receptor 4 by plasma from morphine-treated mice. Brain Behav Immun. (2017) 61:244-58. doi: 10.1016/j.bbi.2016.12.002

81. AlJanahi AA, Danielsen M, Dunbar CE. An Introduction to the analysis of single-cell RNA-sequencing data. Mol Ther Methods Clin Dev. (2018) 10:189-96. doi: 10.1016/j.omtm.2018.07.003

82. Ransohoff RM. A polarizing question: do M1 and M2 microglia exist? Nat Neurosci. (2016) 19:987-91. doi: 10.1038/nn.4338

83. Liu S, Yao JL, Wan XX, Song ZJ, Miao S, Zhao Y, et al. Sonic hedgehog signaling in spinal cord contributes to morphine-induced hyperalgesia and tolerance through upregulating brain-derived neurotrophic factor expression. J Pain Res. (2018) 11:649-59. doi: 10.2147/JPR.S1 53544

84. Hayashi Y, Morinaga S, Zhang J, Satoh Y, Meredith AL, Nakata T, et al. BK channels in microglia are required for morphine-induced hyperalgesia. Nat Commun. (2016) 7:11697. doi: 10.1038/ncomms11697

85. Melik Parsadaniantz S, Rivat C, Rostene W, Reaux-Le Goazigo A. Opioid and chemokine receptor crosstalk: a promising target for pain therapy? Nat Rev Neurosci. (2015) 16:69-78. doi: 10.1038/nrn3858

86. Burma NE, Bonin RP, Leduc-Pessah H, Baimel C, Cairncross ZF, Mousseau M, et al. Blocking microglial pannexin-1 channels alleviates morphine withdrawal in rodents. Nat Med. (2017) 23:355-60. doi: 10.1038/ nm. 4281

87. Burma NE, Leduc-Pessah H, Trang T. Genetic deletion of microglial Panx1 attenuates morphine withdrawal, but not analgesic tolerance or hyperalgesia in mice. Channels (2017) 11:487-94. doi: 10.1080/19336950.2017.1359361

88. Taylor AM, Mehrabani S, Liu S, Taylor AJ, CM. C. Topography of microglial activation in sensory- and affect-related brain regions in chronic pain. $J$ Neurosci Res. (2017) 95:1330-5. doi: 10.1002/jnr.23883

89. Taylor AM, Castonguay A, Taylor AJ, Murphy NP, Ghogha A, Cook C, et al. Microglia disrupt mesolimbic reward circuitry in chronic pain. J Neurosci. (2015) 35:8442-50. doi: 10.1523/JNEUROSCI.4036-14.2015 
90. Taylor AM, Castonguay A, Ghogha A, Vayssiere P, Pradhan AA, Xue L, et al. Neuroimmune regulation of GABAergic neurons within the ventral tegmental area during withdrawal from chronic morphine. Neuropsychopharmacology (2016) 41:949-59. doi: 10.1038/npp.2015.221

91. Cahill CM, Taylor AM. Neuroinflammation-a co-occurring phenomenon linking chronic pain and opioid dependence. Curr Opin Behav Sci. (2017) 13:171-7. doi: 10.1016/j.cobeha.2016.12.003

92. Cahill CM, Walwyn W, Taylor AMW, Pradhan AAA, Evans CJ. Allostatic mechanisms of opioid tolerance beyond desensitization and downregulation. Trends Pharmacol Sci. (2016) 37:963-76. doi: 10.1016/j.tips.2016.08.002

93. Siuda ER, Copits BA, Schmidt MJ, Baird MA, Al-Hasani R, Planer WJ, et al. Spatiotemporal control of opioid signaling and behavior. Neuron (2015) 86:923-35. doi: 10.1016/j.neuron.2015.03.066

94. Madariaga-Mazon A, Marmolejo-Valencia AF, Li Y, Toll L, Houghten RA, Martinez-Mayorga K. Mu-Opioid receptor biased ligands: A safer and painless discovery of analgesics? Drug Discov Today (2017) 22:1719-29. doi: 10.1016/j.drudis.2017.07.002

95. Valentino RJ, Volkow ND. Untangling the complexity of opioid receptor function. Neuropsychopharmacology (2018) 43:2514-20. doi: 10.1038/s41386-018-0225-3

96. Stoeber M, Jullie D, Lobingier BT, Laeremans T, Steyaert J, Schiller PW, et al. A genetically encoded biosensor reveals location bias of opioid drug action. Neuron (2018) 98:963-76.e5. doi: 10.1016/j.neuron.2018. 04.021

97. Doyle HH, Eidson LN, Sinkiewicz DM, Murphy AZ. Sex differences in microglia activity within the periaqueductal gray of the rat: a potential mechanism driving the dimorphic effects of morphine. J Neurosci. (2017) 37:3202-14. doi: 10.1523/JNEUROSCI.2906-16.2017

98. Mapplebeck JCS, Dalgarno R, Tu Y, Moriarty O, Beggs S, Kwok CHT, et al. Microglial P2X4R-evoked pain hypersensitivity is sexually dimorphic in rats. Pain (2018) 159:1752-63. doi: 10.1097/j.pain.0000000000001265

99. Berrettini W. A brief review of the genetics and pharmacogenetics of opioid use disorders. Dialogues Clin Neurosci. (2017) 19:229-36.

100. Grace PM, Hutchinson MR, Maier SF, Watkins LR. Pathological pain and the neuroimmune interface. Nat Rev Immunol. (2014) 14:217-31. doi: $10.1038 /$ nri3621

101. Helmerhorst GT, Teunis T, Janssen SJ, Ring D. An epidemic of the use, misuse and overdose of opioids and deaths due to overdose, in the United States and Canada: is Europe next? Bone Joint J. (2017) 99-B:856-64. doi: 10.1302/0301-620X.99B7.BJJ-2016-1350.R1

Conflict of Interest Statement: The authors declare that the research was conducted in the absence of any commercial or financial relationships that could be construed as a potential conflict of interest.

Copyright (C) 2019 Maduna, Audouard, Dembélé, Mouzaoui, Reiss, Massotte and Gaveriaux-Ruff. This is an open-access article distributed under the terms of the Creative Commons Attribution License (CC BY). The use, distribution or reproduction in other forums is permitted, provided the original author(s) and the copyright owner(s) are credited and that the original publication in this journal is cited, in accordance with accepted academic practice. No use, distribution or reproduction is permitted which does not comply with these terms. 